\title{
I limiti oggettivi del ne bis in idem in Italia tra fonti nazionali ed europee
}

The objective limits of the ne bis in idem in
Italy between national and European rules

Os limites objetivos do ne bis in idem na Itália entre fontes nacionais e europeias

\section{Fabio Salvatore Cassibba ${ }^{1}$}

Università degli Studi di Parma - Parma/Italia fabiosalvatore.cassibba@unipr.it http://lattes.cnpq.br/4632998127399040

https://orcid.org/0000-0002-8971-8064

RiAsSUnto: È assunto consolidato che il ne bis in idem costituisca un diritto fondamentale, protetto da una pluralità di fonti nazionali (art. 649 c.p.p.) e sovranazionali europee (art. 50 CDFUE e 4 prot. n. 7 CEDU). Sennonché, proprio la "tutela multilivello" dei diritti fondamentali che connota l'ordinamento italiano rischia - paradossalmente - di trasformarsi in un ostacolo alla più ampia operatività della garanzia. Le previsioni che individuano i presupposti della garanzia sul piano nazionale e su quello europeo si caratterizzano per un contenuto eterogeneo (identità del fatto; identità dell'infrazione), destinato ad incidere negativamente sull'individuazione della portata oggettiva del divieto di secondo giudizio. D'altra parte, l'interpretazione delle medesime previsioni da parte della giurisprudenza nazionale, ordinaria e costituzionale, e delle Corti sovranazionali rende ancor più incerti i confini dell'effetto preclusivo generato da una decisione irrevocabile. Si allude, in modo particolare, alle ipotesi in cui il ne bis in idem venga in gioco in relazione al "doppio binario sanzionatorio", in forza

1 Professore Associato di Diritto processuale penale - Università degli Studi di Parma. 
del quale un medesimo fatto viene sanzionato, al contempo, sul terreno penale e su quello amministrativo. La ricostruzione della portata di garanzia assegnata all'art. 649 c.p.p. deve muovere dalla valorizzazione del principio di legalità processuale e implica, dunque, da un lato, di ricondurre a unità il presupposto rappresentato dalla medesimezza dell'oggetto dei diversi procedimenti; dall'altro, di scongiurare soluzioni casistiche, contrarie al principio di legalità, in rapporto al presupposto della duplicazione dei diversi procedimenti aventi il medesimo oggetto.

Parole-chiave: Processo penale italiano; fatto nel processo penale; giudicato; ne bis in idem.

AвSTRACT: It is sure that the ne bis in idem constitutes a fundamental right, protected by a plurality of national (article 649 of the Italian Criminal Procedure Code) and European rules (article 50 CDFUE and article 4 protocol n. 7 ECHR). However, precisely the "multilevel protection" of fundamental rights that characterizes the Italian system risks - paradoxically - to become an obstacle to the wider operation of the guarantee. The provisions that identify the content of the double jeopardy both at national and European level are based upon different concept (identity of the fact; identity of the offence), with a negative effect about the identification of the objective limits of the ne bis in idem. At the same time, the interpretation of the same provisions by the national judges, ordinary and constitutional, and by the Supranational Courts makes the boundaries of the ne bis in idem generated by a final decision even more uncertain. We refer to the hypotheses in which the ne bis in idem is linked with "parallel proceedings", where the same fact is sanctioned both by penal and administrative dispositions. The reconstruction of the guarantee provided for by the art. 649 of the Italian Criminal Procedure Code shall strengthen the legality principle and the implies, therefore, on one hand, to reconsider the sameness of the object of the different proceedings; on the other hand, to avoid casuistic solutions, in contrast with to the legality principle, about the duplication of the different proceedings having the same object.

KEY-wORDS: Italian Criminal procedure; factual basis in Italian criminal trial; final decision; double-jeopardy.

Resumo: É premissa consolidada que o ne bis in idem constitua um direito fundamental, protegido por uma pluralidade de fontes nacionais (art. 649 do c.p.p. italiano) e supranacionais (art. 50 CDFUE 
e $4^{\circ}$ protocolo $\mathrm{CEDH}$ ). Entretanto, exatamente a "tutela multinível" dos direitos fundamentais que caracteriza o ordenamento italiano paradoxalmente - corre o risco de se transformar em um obstáculo à mais ampla operabilidade dessa garantia. Ademais, nos panoramas italiano e europeu, as previsões que especificam os pressupostos para a aplicabilidade dessa garantia se caracterizam por um conteúdo heterogêneo (identidade do fato; identidade da infração), destinado a incidir negativamente sobre a definição da perspectiva objetiva da proibição de dupla persecução. Por outro lado, a interpretação das mesmas previsões por parte da jurisprudência italiana, ordinária e constitucional, e pelas Cortes supranacionais torna ainda mais incertos os confins do efeito preclusivo gerado por uma decisão irrevogável. Em particular, alude-se as hipóteses em que o ne bis in idem entre em jogo em relação ao "duplo binário sancionatório", por força do qual o mesmo fato é sancionado, contemporaneamente, em campo penal e em campo administrativo. A reconstrução da perspectiva da garantia atribuída ao art. 649 do c.p.p. italiano deve partir da valorização do princípio de legalidade processual e implica, portanto, por um lado, a recondução da unidade do pressuposto representado pelo mesmo objeto dos diferentes procedimentos; por outro lado, evitar soluções casuísticas, contrárias ao princípio de legalidade, em relação ao pressuposto da duplicação dos diferentes procedimentos que se refiram ao mesmo fato.

Palavras-chave: Processo penal italiano; fato no processo penal; trânsito em julgado; ne bis in idem.

Sommario: 1. Il Ne bis in idem: un diritto fondamentale dai malsicuri confini normativi. 2. La coincidenza fra disciplina nazionale e sovranazionale circa l'identità del fatto. 2.1. Identità del fatto e ne bis in idem nelle fonti sovranazionali. 2.2. Identità del fatto e ne bis in idem nel codice di procedura penale italiano. 2.3. Le prassi elusive del ne bis in idem nella giurisprudenza nazionale e la reazione della Corte costituzionale. 3. La "nuova frontiera": ne bis in idem e doppio binario sanzionatorio. 3.1 Ne bis in idem e doppio binario sanzionatorio nelle fonti sovranazionali. 3.2. $\mathrm{Ne}$ bis in idem e doppio binario sanzionatorio nell'ordinamento nazionale. Bibliografia. 


\section{Il Ne bIS IN IDEM: UN DIRITTO FONDAMENTALE DAI MALSICURI CONFINI NORMATIVI}

La garanzia del ne bis in idem ${ }^{2}$ sconta nell'ordinamento nazionale gli effetti negativi d'un paradosso: considerata dalla dottrina ${ }^{3} \mathrm{e}$ dalla più recente giurisprudenza alla stregua d'un diritto fondamentale ${ }^{4}$, resta condizionata da malsicuri confini normativi. Alla base dell'incertezza sta il sistema di "tutela multilivello" dei diritti fondamentali, proprio dell'ordinamento italiano ${ }^{5}$, caratterizzato da un labirintico intreccio delle

2 Sul tema la letteratura è vastissima: fatti salvi gli ulteriori richiami nel corso del presente, fra i lavori più recenti, cfr., anche per il rinvio agli ampi apparati bibliografici, BONTEMPELLI, Manfredi. La litispendenza penale, Giuffrè, 2017, spec. p. 52 s.; CAPRIOLI, Francesco. Il principio del ne bis in idem, in CAPRIOLI, Francesco; VICOLI, Daniele. Procedura penale dell'esecuzione, Torino, 2011, p. 67 s., CORDERO, Franco. Procedura penale, Giuffrè, 2012, p. 1201 s.; MANCUSO, Enrico Maria, Il giudicato nel processo penale, in UBERTIS, Giulio, VOENA; Giovanni Paolo (diretto da). Trattato di procedura penale, XLI.1, Giuffrè, 2012, p. 42 ss.; RAFARACI, Tommaso. Ne bis in idem, in Enc. dir., Annali, III, Giuffrè, 2010, p. 857 ss.

3 Cfr. GALANTINI, Novella. Il divieto del doppio processo per lo stesso fatto come diritto della persona, in Riv. it. dir. proc. pen., 1981, p. 101 ss.; RAFARACI, Tommaso. Ne bis in idem, cit., p. 858.

4 Così, fra le molte, C. giust. UE, grande camera, 26 febbraio 2013, C-617/10, Åklagaren c. Hans Åkerberg Fransson, § 16 ss.; v. anche C. giust. UE, grande camera, 27 maggio 2014, C-129/14 PPU, Zoran Spasic, § 51 ss. Dal punto di vista interno, che il ne bis in idem integri un diritto fondamentale a tutela dell'imputato è espressamente affermato, in rapporto all'art. 50 CDFUE, da Cass., sez. VI, 15 novembre 2016, Resnelli, in Dir. pen. cont., ed. on-line, 7 aprile 2017 , p. 8 , con nota di I. Gittardi, disponibile in http://www.penalecontemporaneo.it, ultimo accesso il 24 agosto 2018. Dal canto suo, la Corte costituzionale da tempo considera il ne bis in idem come un «principio di civiltà giuridica»: cfr. C. cost., ord. 5 maggio 1995 n. 150, in GU, I serie speciale, n. 19 del 10 maggio 1995, disponibile all'indirizzo www.gazzettaufficile.it; da ultimo, C. cost., sent. 21 luglio 2016 n. 200, in Cass. pen., 2017, p. 60 ss.

5 Sul tema, fra i numerosi contributi, BUZZELLI, Silvia. Processo penale europeo, in Enc. dir., Annali, II, tomo I, Giuffrè, 2008, p. 701 ss.; CAPRIOLI, Francesco. Giudicato e illegalità della pena: riflessioni a margine di una recente sentenza della Corte costituzionale, in BARGIS, Marta (a cura di). Studi in ricordo di Maria Gabriella Aimonetto, Giuffrè, 2013, p. 263 ss.; CASSESE, Sabino. I tribunali di Babele. I giudici alla ricerca di un nuovo ordine globale, Donzelli, 2009, p. 1 ss.; DANIELE, Marcello. La triangolazione delle garanzie processuali tra Diritto dell'Unione europea, Cedu e sistemi nazionali, in Dir. pen. cont., ed. on-line del 6 aprile 2016, p. 1 ss., disponibile in <http://www.penalecontemporaneo.it>, 
fonti nazionali e sovranazionali che regolano la materia ${ }^{6}$, per di più, fra loro contenutisticamente eterogenee e fondate su vocaboli a basso coefficiente di determinatezza.

Quanto ai presupposti della garanzia, pesa l'ambiguità semantica delle previsioni nazionali e sovranazionali. Il ne bis in idem è ancorato dall'art. 649 comma 1 c.p.p. alla medesimezza del «fatto» ${ }^{7}$; dagli art. 4 prot. n. 7 CEDU e 50 CDFUE (come, del pari, dall'art. 14 comma 7 PIDU) alla medesimezza dell'«infraction» o dell'«offence» ${ }^{8}$. Così, sulla base del dato letterale, la norma nazionale riveste una portata garantistica maggiore di quelle sovranazionali, perché vanta una sfera operativa più estesa: comunque lo si voglia intendere, il concetto di medesimo «fatto» appare più ampio di quello di «offesa» $\mathrm{O}$ «infrazione», che riveste con una qualificazione normativa il "nudo fatto" oggetto del giudizio.

Quanto all'effetto preclusivo, le fonti nazionali e sovranazionali la regolano in modo diverso. L'art. 649 comma 1 c.p.p. preclude il nuovo esercizio dell'azione penale per lo stesso fatto nei confronti del soggetto già irrevocabilmente prosciolto o condannato, indipendentemente dall'esito

ultimo accesso il 24 agosto 2018; DINACCI, Filippo Raffale. Interpretazione "europeisticamente" orientata tra fonti normative e resistenze giurisprudenziali, in GAITO, Alfredo (a cura di). I princìpi europei del processo penale, cit., p. 58 ss.; DI GIOVINE, Ombretta, Come la legalità europea sta riscrivendo quella nazionale. Dal primato della legge a quello dell'interpretazione, in Dir. pen. cont. Riv. trim., 2013, fasc. 1, p. 159 ss.; GALLIANI, Davide. Sul mestiere del giudice, tra Costituzione e Convenzione, disponibile in <www.giurcost.org $>$, ultimo accesso il 24 agosto 2018; GAMBINI, Rosanna. Armonizzazione dei diritti nazionali nel segno della giurisprudenza europea, in Dir. pen. proc., 2009, p 1169 ss.; KOSTORIS, Roberto-Ettore. La tutela dei diritti fondamentali, in KOSTORIS, Roberto-Ettore (a cura di), Manuale di procedura penale europea, Giappichelli, 2017, p. 84 ss. MANES, Vittorio. Il giudice nel labirinto. Profili delle intersezioni tra diritto penale e fonti sovranazionali, Dike, 2012, p. 135 ss.; MAZZA, Oliviero. Giustizia penale in trasformazione: profili d'indagine, in Arch. pen., 2012, fasc. 1, p. 17 ss.; UBERTIS, Giulio. Sistema multilivello dei diritti fondamentali e prospettiva abolizionista del processo contumaciale contumaciale (2009), in Id., Argomenti di procedura penale, vol. III, Giuffrè, 2011, p. 185 ss.

6 Per riprendere la felice metafora di MANES, Vittorio. Il giudice nel labirinto. Profili delle intersezioni tra diritto penale e fonti sovranazionali, cit.

7 Sulla polisemia del vocabolo nel lessico processual-penalistico, v. infra, § 2 .

8 Suquesti profili e sull'interpretazione offerta dalla giurisprudenza sovranazionale in materia, v. infra, $\S 2$. 
dell'ipotetico secondo giudizio. Gli art. 4 prot. n. 7 CEDU e 50 CDFUE lungi dall'escludere la riprosecution per il medesimo reato - contemplano un duplice, alternativo effetto preclusivo: il già prosciolto o condannato non può essere nuovamente «perseguito o condannato» per quel reato. In altri termini, in forza delle norme europee la garanzia del ne bis in idemnon vieta affatto la doppia decisione, invece sempre vietata dall'art. 649 comma 1 c.p.p. ${ }^{9}$, quando il secondo epilogo abbia esito favorevole per l'imputato. Così, rispetto a quanto appena evidenziato in ordine ai presupposti nazionali e sovranazionali della garanzia, qui i termini s'invertono: sulla base del dato letterale sono gli art. 4 prot. n. 7 CEDU e 50 CDFUE a rivestire un significato maggiormente garantista rispetto all'art. 649 comma 1 c.p.p. ${ }^{10}$. Se è vero che quest'ultimo anticipa l'operatività della garanzia, precludendo il secondo processo, è altrettanto vero che, così facendo, esclude a priori l'epilogo ampiamente favorevole all'imputato all'esito del secondo processo, che, a sua volta, potrebbe costituire titolo per la revoca del giudicato di condanna ai sensi dell'art. 669 c.p.p.

V'è di più. La nuova frontiera della garanzia del ne bis in idem il cui confine è oltremodo incerto - è, oggi, rappresentata dal rapporto fra il divieto di secondo giudizio e il "doppio binario sanzionatorio", operante quando lo stesso fatto è sanzionato, al contempo, dalla legge penale e dalla legge amministrativa ${ }^{11}$. Emerge, qui, un sensibile contrasto

9 Cfr. MANCUSO, Enrico Maria. Art. 4 prot. 7 - Divieto di secondo giudizio, in UBERTIS, Giulio; VIGANÒ, Francesco (diretto da). Corte di Strasburgo e giustizia penale, Giappichelli, 2016, p. 374 ss.

10 Così, MAZZA, Oliviero. L'insostenibile convivenza fra ne bis in idem europeo e doppio binario sanzionatorio per i reati tributari, in Rass. Trib., 2015, n. 4, p. 1035, la cui ricostruzione è ripresa da BONTEMPELLI, Manfredi. La litispendenza penale, cit., p. 64-65. V., pure, FALCINELLI, Daniela, Il fatto di reato sullo sfondo del ne bis in idem nazional-europeo, in Arch. pen., 2017, fasc. 1, spec. p. 69.

11 Sul tema la letteratura è amplissima: cfr., senza pretesa di completezza, e fatti salvi gli ulteriori riferimenti nel corso del presente lavoro, AMALFITANO, Chiara, D’AMBROSIO, Raffaele. Art. 50 - Diritto a non essere giudicato o punito due volte per lo stesso reato, in MASTROIANNI, Roberto, POLLICINO, Oreste, ALLEGREZZA, Silvia, PAPPALARDO, Fabio, RAZZOLINI, Orsola (a cura di). Carta dei diritti fondamentali dell'Unione europea, Milano, 2017, p. 1031 ss.; BONTEMPELLI, Manfredi. La litispendenza penale, Milano, 2017, p. 64 ss. e 201 ss.; Id., Ne bis in idem e legalità penale nel processo per gli abusi di mercato, in Arch. pen., 2016, n. 2, p. 389 ss.; CAIANIELLO, Michele. $\mathrm{Ne}$ bis in idem e illeciti tributari per omesso versamento dell'Iva: il rinvio 
fra l'ordinamento nazionale e quelli sovranazionali. Da un lato, mentre in forza dell'art. 649 c.p.p. solo un provvedimento formalmente penale, passato in giudicato, è suscettibile di sprigionare l'effetto preclusivo in ordine al fatto oggetto del giudizio, gli art. 50 CDFUE e 4 prot. n. 7 CEDU - nell'interpretazione datane dalla Corte di giustizia e dalla Corte europea $^{12}$ - assegnano l'identico effetto preclusivo anche ad un provvedimento formalmente pronunciato da un'autorità amministrativa ma avente

della questione alla Corte costituzionale, in Dir. pen. cont., ed. on-line del 15 maggio 2015, p. 1 ss., disponibile in http://www.penalecontemporaneo.it, ultimo accesso il 24 agosto 2018; CASSIBBA, Fabio. Disorientamenti giurisprudenziali in tema di ne bis in idem e procedimenti paralleli, in Proc. pen. giust., 2017, fasc. 6, p. 1098 ss.; CORSO, Piermaria. Prospettive evolutive del ne bis in idem, in Arch. pen., 2017, fasc. 1, p. 13 ss.; DI BITONTO, Maria Lucia. Una singolare applicazione dell'art. 649 c.p.p., in Dir. pen. proc., 2015, p. 441 ss.; DOVA, Massimiliano. Ne bis in idem e reati tributari: una questione ormai ineludibile, in Dir. pen. cont., ed. on-line dell'11 dicembre 2014, disponibile in http://www.penalecontemporaneo.it, ultimo accesso il 24 agosto 2018; GALATINI, Novella. Il principio del ne bis in idem tra doppio processo e doppia sanzione, in Giur. it., 2015, p. 217 ss.; Ead., Postilla ad uno scritto in tema di ne bis in idem, in Dir. pen. cont., ed. on-line del 30 gennaio 2017, p. 1 ss., disponibile in http://www.penalecontemporaneo.it, ultimo accesso il 24 agosto 2018; Ead., Il 'fatto' nella prospettiva del divieto di secondo giudizio, in Riv. it. dir. proc. pen., 2017, p. 1205 ss.; MANACORDA, Stefano. Equazioni complesse: il ne bis in idem "ancipite" sul "doppio binario" per gli abusi di mercato al vaglio della giurisprudenza, in Dir. pen. proc., 2017, p. 515 ss.; MANCUSO, Enrico Maria. Ne bis in idem e giustizia sovranazionale, in MANCUSO, Enrico Maria. Ne bis in idem e giustizia sovranazionale, GIARDA, Angelo; PERINI, Andrea; VARRASO, Gianluca (a cura di). La nuova giustizia penale tributaria, Padova: Cedam, 2016, p. 533 ss. La nuova giustizia penale tributaria, Padova, 2016, p. 533 ss.; MAZZA, Oliviero. L'insostenibile convivenza fra ne bis in idem europeo e doppio binario sanzionatorio per $i$ reati tributari, cit., p. 1033 ss.; NASCIMBENE, Bruno. Ne bis in idem, diritto internazionale, diritto europeo, in Eurojus, ed. on-line del 22 marzo 2018, disponibile in http://www.eurojus.it, ultimo accesso il 24 agosto 2018; PAULESU, Pier Paolo. Ne bis in idem e conflitti di giurisdizione, in KOSTORIS, Roberto Ettore (a cura di). Manuale di procedura penale europea, cit., p. 478 ss.; RIVELLO, Pier Paolo. I rapporti tra giudizio penale e tributario ed il rispetto del principio del ne bis in idem, in Dir. pen. cont., 2018, fasc. 1, p. 101 ss., disponibile in http://www.penalecontemporaneo.it, ultimo accesso il 24 agosto 2018; VIGANÖ, Francesco. Art. 4 Prot. $n .7$ - Diritto a non essere giudicato o punito due volte, in UBERTIS, Giulio; VIGANÒ, Francesco (a cura di), Corte di Strasburgo e giustizia penale, cit., p. 383 ss. 
natura "sostanzialmente" penale, in forza degli "Engel criteria", volti, per l'appunto, a delimitare la "matière pénale"13.

In prospettiva generale, la complessità e il diverso contenuto delle fonti che regolano il divieto di secondo giudizio amplificano la discrezionalità interpretativa del giudice, indebolendone la soggezione soltanto alla legge e determinando l'aggiramento del canone della legalità processuale, reclamati dagli art. 101 comma 2 e 111 comma 1 Cost. Come si vedrà, interpretazioni antiletterali dell'art. 649 comma 1 c.p.p., da un lato, e degli art. 4 prot. n. 7 CEDU e 50 CDFUE, dall'altro, sono state fornite tanto dalla giurisprudenza nazionale quanto da quella sovranazionale ${ }^{14}$.

Dal punto di vista dell'ordinamento nazionale, emerge la «metamorfosi in atto [nei] rapporti fra giudice e legge penale ${ }^{15}$, con il rischio di «consegn[are nelle mani del primo] le chiavi della legalità processuale» ${ }^{16}$ :

13 Così definiti perché elaborati, per la prima volta, da C. eur. dir. uomo, sent. 8 giugno 1976, Engel c. Paesi Bassi e mai più messi in discussione. Sul tema, infra, § 3 .

14 V. infra, § 2.

15 CAPRIOLI, Francesco. Giudicato e illegalità della pena: riflessioni a margine di una recente sentenza della Corte costituzionale, cit., p. 263.

16 MAZZA, Oliviero. La Corte assediata e il garantismo efficiente (note a margine della Carta di Napoli), in Proc. pen. giust., 2014, fasc. 5, p. 8. Sulla crisi della legalità processuale nel sistema italiano, anche per opera delle suggestioni offerte dalla giurisprudenza sovranazionale, cfr., fra i molti, LORUSSO, Sergio. Interpretazione, legalità processuale e convincimento del giudice, in Dir. pen. cont., ed. on-line del 10 giugno 2015, disponibile in <http://www.penalecontemporaneo.it>, ultimo accesso il 24 agosto 2018; MAZZA, Oliviero. Il pregiudizio effettivo fra legalità processuale e discrezionalità del giudice, in Giust. pen., 2015, III, c. 697 ss.; Id., L'irruzione delle corti sovranazionali, Aa.Vv., Legge e potere nel processo penale. Pensando a Massimo Nobili, Cedam, 2017, p. 182 ss.; Id., Ideologie della riforma Orlando, in TESTAGUZZA, Alessandra (a cura di). Esercitazioni penali sostanziali e processuali. Pensieri in ordine sparso, Cedam, 2018, p. 229; NEGRI, Daniele. Splendori e miserie della legalità processuale. Genealogie culturali, èthos delle fonti, dialettica tra le Corti, ivi, p. 43 s.; UBERTIS, Giulio. Equità e proporzionalità versus legalità processuale: eterogenesi dei fini, in Arch. pen., 2017, n. 2, p. 389 ss. Più in generale, v., già, AMODIO, Ennio. Crisi della legalità processuale, filosofia della rassegnazione e autorevolezza dei giuristi, in Riv. it. dir. proc. pen., 2004, p. 432 ss.; GALANTINI, Novella. Considerazioni su principio di legalità processuale, in Cass. pen., 1999, p. 1989 ss.; NOBILI, Massimo. Principio di legalità, processo, diritto sostanziale (1995), in Scenari e trasformazioni del processo penale, Padova, 1998, p. 181 ss.; PADOVANI, Tullio. Il crepuscolo della legalità nel 
il "diritto vivente" prevale vieppiù su quello "vigente"17, rovesciando il classico «paradigma» della primazia della legge formale sul formante giurisprudenziale $^{18}$. Più precisamente, il dovere di osservare i vincoli derivanti dagli obblighi internazionali - imposto dall'art. 117 comma 1 Cost. anzitutto al legislatore ma pure ai giudici comuni - fa sì che gli operatori siano alla spasmodica ricerca di un significato dell'art. 649 c.p.p. coerente, al contempo, con i canoni costituzionali ${ }^{19}$, con quelli convenzionali e con quelli del diritto dell'Unione europea.

La faticosa tessitura della tela di Penelope da parte dei giudici nazionali, volta a rivestire di un univoco contenuto la garanzia del ne bis in idem, comporta una pesante contropartita in rapporto alla certezza del diritto e delle situazioni giuridiche soggettive. Il divieto di secondo giudizio - in linea di principio, destinato ad una sicura espansione sistematica, proprio in virtù della sua natura di diritto fondamentale ${ }^{20}$ - risulta

processo penale. Riflessioni antistoriche sulle dimensioni processuali della legalità penale, in Ind. pen., 1999, p. 527 ss.

17 FERRUA, Paolo. Soggezione del giudice alla sola legge e disfunzioni del legislatore: il corto circuito della riforma Orlando, in Dir. pen. proc., 2017, p. 1265 ss.

18 Così, CAPRIOLI, Francesco. Il giudice e la legge processuale: il paradigma rovesciato, in Ind. pen., 2017, p. 967 ss.

19 Benché difetti di un espresso riconoscimento da parte della Carta costituzionale, sulla copertura costituzionale del ne bis in idem concorda, pur con vari accenti, la dottrina: v., anche per ulteriori riferimenti, BONTEMPELLI, Manfredi. La litispendenza penale, cit., p. 55 ss.; CAPRIOLI, Francesco. Il principio del ne bis in idem, cit., p. 73 ss.; MANCUSO, Enrico Maria. Il giudicato nel processo penale, cit., p. 431 ss. Nella giurisprudenza costituzionale, da ultimo, C. cost., sent. 21 luglio 2016 n. 200, cit., p. 60 ss.

20 In generale, cfr. i vari contributi raccolti in FERRAJOLI, Luigi. Diritti fondamentali. Un dibattito teorico, a cura di VITALE, Ermano, Bari: Laterza, 2008, passim.

Da altro punto di vista, merita, poi, ricordare che le Sezioni unite hanno - dopo aver ravvisato nel ne bis in idem un principio generale dell'ordinamento - ne hanno esteso la sfera lungo tutto l'arco del procedimento, anche prima del formarsi del giudicato, a condizione che proceda il medesimo ufficio del pubblico ministero: cfr. Cass., sez. un., 28 giugno 2005, Donati ed altro, in Cass. pen., 2006, p. 28 ss., sulla quale v., altresì, MONTAGNA, Mariangela. Ne bis in idem e sentenza non irrevocabile: si allarga l'ambito della preclusione, in Dir. pen. proc., 2005, p. 134 ss.; più di recente, CONTI, Carlotta. La preclusione nel processo penale, Giuffrè, 2014, p. 74 ss.; MANCUSO, Enrico Maria. Il giudicato nel processo penale, cit., spec. p. 456 ss. Nel senso, invece, che l'art. 649 c.p.p., letto in 
sempre esposto a ridefinizioni. Da qui, un triplice rischio: che il ne bis in idem non si risolva in un diritto concreto ed effettivo bensì resti teorico ed illusorio ${ }^{21}$; che la sua portata non sia prevedibile dai consociati ${ }^{22}$; che i titolari del diritto fondamentale non siano al riparo da disparità di trattamento per opera della prassi.

\section{LA COINCIDENZA FRA DISCIPLINA NAZIONALE E SOVRANAZIONALE CIRCA L'IDENTITÀ DEL FATTO}

A dispetto dell'appena evidenziata eterogeneità contenutistica fra l'art. 649 c.p.p., da un lato, e gli art. 4 prot. 7 CEDU e 50 CDFUE, dall'altro, i sistemi di tutela nazionale e sovranazionali mostrano una significativa coincidenza in ordine ai presupposti del ne bis in idem, frutto d'una travagliata elaborazione giurisprudenziale.

\subsection{IDENTITÀ DEL FATTO E NE BIS IN IDEM NELLE FONTI SOVRANAZIONALI}

Benché gli art. 4 prot. 7 CEDU e 50 CDFUE fondino il divieto di secondo giudizio sul presupposto della medesimezza dell'offesa o dell'infrazione, è significativo che la giurisprudenza della Corte di Giustizia e della Corte europea dei diritti dell'uomo abbiano operato una radicale ridefinizione della portata delle previsioni in parola. Superando il dato testuale, i giudici di Strasburgo e di Lussemburgo sono pervenuti ad una concezione del divieto ancorata alla medesimezza del fatto ${ }^{23}$.

chiave sistematica, non impedisce che un secondo processo possa validamente sorgere prima che intervenga il giudicato nel primo, RAFARACI, Tommaso. Le nuove contestazioni nel processo penale, Giuffrè, 1996, spec. p. 273.

21 Per impiegare un'affermazione assolutamente consolidata nel case-law della Corte europea dei diritti dell'uomo: per tutte, fra le moltissime, con riguardo ad una vicenda di grande rilievo, anche mediatico, C. eur. dir. uomo, sez. IV, sent. 7 luglio 2015, Cestaro c. Italia, § 207 ss.

22 Benché la prevedibilità della portata della garanzia costituisca l'oggetto della consolidata giurisprudenza di Strasburgo: cfr., per tutte, C. eur. dir. uomo, grande camera, 15 novembre 2016, A e B c. Norvegia, § 130.

23 L'evoluzione della giurisprudenza della Corte europea è evidenziata, fra gli altri, da DE AMICIS, Gaetano. Ne bis in idem e "doppio binario" sanzionatorio: 
Premesso che la concisione del dato letterale non permette di comprendere con certezza se in sede di redazione dell'art. 4 prot. n. 7 CEDU si fosse inteso dare rilievo alla qualifica giuridica del fatto o alla sola nozione naturalistica dell'idem factum $^{24}$, l'approccio tipicamente casistico e pragmatico della Corte europea, fondato sulla valorizzazione delle peculiarità della vicenda, ha comportato, per lungo tempo, significative oscillazioni nella giurisprudenza di Strasburgo ${ }^{25}$.

Per un primo indirizzo, la nozione di «infraction» o di «offence» che compare nell'art. 4 prot. 7 CEDU va intesa in senso formale, ossia secondo un'accezione che appare maggiormente aderente al tenore della previsione. La riprosecution non è esclusa quando, pur in presenza della medesima condotta, quest'ultima sia capace di violare diverse fattispecie incriminatrici, come accade per la figura del concorso formale eterogeneo di reati ${ }^{26}$. Per un secondo indirizzo, il presupposto è suscettibile di una più ampia lettura, animata dallo scopo di non marginalizzare la garanzia del ne bis in idem: rileva l'identità del fatto materiale ${ }^{27}$, a sua volta apertamente

prime riflessioni sugli effetti della sentenza “Grande Stevens" nell'ordinamento italiano, in Dir. pen. cont. - Riv. trim., 2014, fasc. 3-4, p. 203, disponibile in http://www.penalecontemporaneo.it, ultimo accesso il 24 agosto 2018, e da FERRUA, Paolo. La sentenza costituzionale sul caso Eternit: il ne bis in idem tra diritto vigente e diritto vivente, in Cass. pen., 2017, p. 78 ss.

24 In tali precisi termini, MANCUSO, Enrico Maria. Art. 4 prot. 7 - Diritto a non essere giudicato o punito due volte, cit., p. 380.

25 Cfr., per tutti, GALANTINI, Novella. Il 'fatto' nella prospettiva del divieto di secondo giudizio, cit., p. 1212 ss.

26 Cfr., fra le altre, C. eur. dir. uomo, sez. I, sent. 6 giugno 2012, Sailer c. Austria, $\S 25$; sez. III, sent. 29 magio 2001, Franz Fischer c. Austria, § 25; sent. 23 ottobre 1998, Oliveira c. Svizzera, § 27.

27 Il leading case del revirement giurisprudenziale è rappresentato da C. eur. dir. uomo, grande camera, sent. 10 febbraio 2009, Sergey Zolotukhin c. Russia. Da un diverso punto di vista, merita comunque evidenziare che la protezione offerta dall'art 4 prot. 7 CEDU non è assoluta. La Corte europea ha evidenziato che, quando vengano in gioco gravissimi delitti, caratterizzati - sul piano internazionale - dall'imprescrittibilità del reato, il proscioglimento irrevocabile per estinzione del reato sul piano nazionale dovuta al decorso del termine di prescrizione non preclude un secondo giudizio per il medesimo fatto, occorrendo assicurare una tutela effettiva ai diritti fondamentali protetti dagli art. 2 e 3 CEDU (diritto alla vita e divieto di tortura): cfr., con riguardo a crimini di guerra, C. eur. di. uomo, grande camera, sent. 27 maggio 2014, Marguš c. Croazia. Sul tema v. anche C. cost., sent. 21 luglio 2016 n. 200, cit., 
ispirata all'interpretazione già fornita dalla Corte di giustizia a proposito dell'identico tenore dell'art. 50 CDFUE $^{28}$.

La duplice esigenza, da un lato, di procedere a un'«armonizzazione» delle diverse letture offerte dai giudici di Strasburgo a proposito della portata dell'art. 4 comma 1 Prot. n. 7 Conv. eur. dir. uomo ${ }^{29}$, dall'altro, di assicurare una protezione effettiva contro la riprosecution in favore dell'imputato già condannato o prosciolto ${ }^{30}$ è stata, così, al centro di una decisiva pronuncia della grande camera della Corte europea ${ }^{31}$, che - superando ogni residua ambiguità semantica proveniente dall'art. 4 prot.

p. 65; in dottrina, sulla portata dell'art. 649 c.p.p. in rapporto alla pronuncia europea, LAVARINI, Barbara. Il 'fatto' ai fini del ne bis in idem nella legge italiana e nella CEDU: la Corte costituzionale alla ricerca di un difficile equilibrio, in Proc. pen. giust., 2017, fasc. 1, p. 60 ss. Più in generale, sulle implicazioni, sostanziali e processuali, dell'effettiva protezione dei diritti fondamentali in parola, cfr. CASSIBBA, Fabio; COLELLA, Angela. Art. 3 - Proibizione della tortura, in UBERTIS, Giulio; VIGANÒ, Francesco (a cura di). Corte di Strasburgo e giustizia penale, cit., p. 64 ss.

Così, PAULESU, Pier Paolo. Ne bis in idem e conflitti di giurisdizione, cit., p. 416; analogamente, VERVAELE, John. Ne bis in idem: verso un principio costituzionale transnazionale in UE?, in Riv. it. dir. proc. pen., 2014, p. 53; v. anche DELLA MONICA, Giuseppe. Ne bis in idem. In GAITO, Alfredo (a cura di). I principi europei del processo penale, Dike, 2016, p. 331 ss.; BRANCACCIO, Matilde; FIDELBO, Giorgio. Ne bis in idem: percorsi giurisprudenziali e recenti approdi della giurisprudenza nazionale ed europea, Relazione di orientamento dell'Ufficio del Massimario penale, 21 marzo 2017, in www.cortedicassazione. it, p. 13 ss.cit., p. 2 ss.; ZAGREBELSKY, Vladimiro. Le sanzioni Consob, l'equo processo e il ne bis in idem nella C.e.d.u., in Giur. it., 2014, p. 1199 ss. Nella giurisprudenza di Lussemburgo v., fra le altre, C. giust. UE, sez. II, sent. 9 marzo 2006, C-436/04, Léopold Henri Van Esbroek; C. giust. UE, sez. II, sent. 28 settembre 2006, C-150/05, Jan Leo Van Straaten.

Così, C. eur. dir. uomo, grande camera, sent. 10 febbraio 2009, Sergey Zolotukhin c. Russia, § 78. V. anche, C. eur. dir. uomo, sez. II, sent. 27 novembre 2014, Lucky Dev c. Svezia, § 52.

Merita evidenziare che, in almeno una occasione, la Corte europea ha ritenuto che la condanna in sede amministrativa-penale per i medesimi fatti già oggetto di sentenza penale di assoluzione passata in giudicato violasse la presunzione d'innocenza sancita dall'art. 6 comma 2 CEDU: così, C. eur. dir. uomo, sez. I, sent. 30 aprile 2015, Kapetanios e altri c. Grecia, § 49 ss.

31 Cfr., in tal senso, C. eur. dir. uomo, grande camera, sent. 10 febbraio 2009, Zolotukhin c. Russia, § 80; nonché, più di recente, sez. IV, sent. 20 maggio 2014, Pirttimäki c. Finlandia, § 49. 
7 CEDU - ha stabilizzato l'orientamento incentrato sulla concezione naturalistica del fatto rilevante ai fini del ne bis in idem.

L'odierno assetto poggia su due pilastri. Sul piano metodologico, il vaglio circa la medesimezza del fatto non si risolve nel mero confronto fra le accuse contestate nei due procedimenti: l'esposizione del fatto oggetto del processo costituisce solo un «utile punto di partenza» ${ }^{32}$, occorrendo un'analisi scrupolosa sulle specifiche vicende, anche alla luce delle coordinate spaziali e temporali in cui si dipanano le condotte considerate ${ }^{33}$. Sul piano contenutistico, vale un criterio basato strettamente sull'«identità del fatto materiale» ascritto all'imputato nei due procedimenti, senza che rilevi la diversa qualificazione giuridica ${ }^{34}$ : il presupposto è integrato quando i procedimenti abbiano ad oggetto «fatti identici» o un «fatto che sia sostanzialmente il medesimo». In breve, l'identità materiale del fatto è data dalla «medesima condotta» tenuta dall'imputato, nell'identico contesto spaziale e temporale ${ }^{35}$.

32 Cfr. C. eur. dir. uomo, sez. II, sent. 4 marzo 2014, Grande Stevens e altri c. Italia, § 220; grande camera, sent. 10 febbraio 2009, Zolotukhin c. Russia, § 83.

33 Per C. eur. dir. uomo, grande camera, sent. 10 febbraio 2009, Zolotukhin c. Russia, § 82, occorre avere riguardo ad un «complesso di concrete circostanze fattuali relative al medesimo imputato, inscindibilmente avvinte sul piano temporale e spaziale».

34 C. eur. dir. uomo, grande camera, sent. 10 febbraio 2009, Zolotukhin c. Russia, § 79.

35 Così, C. eur. dir. uomo, sez. II, sent. 4 marzo 2014, Grande Stevens e altri c. Italia, § 224 e 227. Da ultimo, cfr. C. eur. dir. uomo, sez. I, sent. 30 aprile 2015, Kapetanios e altri c. Grecia, § 64; sez. IV, 27 gennaio 2015, Rinas c. Finlandia, $\S 45$; sez. II, sent. 27 novembre 2014, Lucky Dev c. Svezia, § 52. Restano, così, superate le «ambiguità della Corte europea ... emergent[i], per un verso, dalla reputata legittimità di una duplice condanna relativa a uno stesso fatto nell'ipotesi di concorso formale di reati con assorbimento della pena più lieve da parte di quella più grave ..., e, per contro, dalla ritenuta violazione del precetto pattizio in un caso in cui il medesimo comportamento aveva originato due contrastanti decisioni attraverso una difforme qualificazione giuridica operata da organi nazionali dissimili» (UBERTIS, Giulio. Principi di procedura penale europea. Le regole del giusto processo, Cortina, 2009, p. 46; v. pure PAULESU, Pier Paolo. Ne bis in idem e conflitti di giurisdizione, cit., p. 349).

Tocca appena notare che, quando all'imputato vengano contestate condotte identiche ma reiterate nel tempo e nello spazio, la garanzia pattizia non opera (cfr., ad esempio, C. eur. dir. uomo, sez. III, sent. 5 ottobre 2006, Marcello Viola c. Italia, $\S 88$ ss.): qui, si è in presenza di una pluralità di episodi 
In una prospettiva sovranazionale ancora diversa merita, infine, evidenziare che l'interpretazione oggi consolidata della Corte di Strasburgo e della Corte di Lussemburgo si pone in linea con il tenore dell'art. 54 CAAS, che basa l'operatività del ne bis in idem internazionale sulla medesimezza dei «fatti» oggetto dei diversi procedimenti, intesa in senso storico-naturalistico, ritenendosi irrilevante la qualifica o il bene giuridico leso ${ }^{36}$.

\subsection{IDENTITÀ DEL FATTO E NE BIS IN IDEM NEL CODICE DI PROCEDURA PENALE ITALIANO}

Come anticipato, l'art. 649 comma 1 c.p.p. condiziona il divieto di secondo giudizio al presupposto della medesimezza del fatto per cui si procede nuovamente contro il medesimo imputato, rispetto a quello già oggetto della sentenza passata in giudicato, con l'ulteriore, espressa precisazione che la diversa considerazione del fatto «per titolo, grado e circostanze» non basta ad escludere il ne bis in idem.

Dunque, la portata della garanzia dipende, anzitutto, dal significato che s'intende assegnare al vocabolo "fatto".

E' agevole intuire che il "fatto" ricopre un ruolo sistematico chiave, intersecando «punti vitali del processo penale» ${ }^{37}$ : nella sua accezione

autonomi, che possono essere contestati cumulativamente nel medesimo processo o autonomamente in separate vicende: cfr., da ultimo, C. eur. dir. uomo, sez. II, sent. 27 novembre 2014, Lucky Dev c. Svezia, § 55; C. eur. dir. uomo, sez. IV, sent. 20 maggio 2014, Pirttimäki c. Finlandia, § 49 ss.

Sul concetto di idem factum ai fini del ne bis in idem internazionale, oltre al contributo monografico di GALANTINI, Novella. Il principio del ne bis in idem internazionale nel processo penale, Milano, 1984, passim, v., fra gli altri, CAPRIOLI, Francesco. Il principio del ne bis in idem, cit., p. 70 ss.; MANCUSO, Enrico Maria. Il giudicato nel processo penale, cit., p. 525 ss.; PISAPIA, Alice. Riflessioni sul principio del ne bis in idem alla luce delle recenti pronunce della Corte di Giustizia dell'Unione europea, in Cass. pen., 2013, spec. p. 3280 ss.; RAFARACI, Tommaso. Ne bis in idem e conflitti di giurisdizione in materia penale nello spazio di libertà, sicurezza e giustizia dell'Unione Europea, in Riv. dir. proc., 2007, p. 625 ss.; VIGANÒ, Francesco. Doppio binario sanzionatorio $e$ ne bis in idem: verso una diretta applicazione dell'art. 50 della Carta?, in Dir. pen. cont. - Riv. trim., 2014, fasc. 3-4, p. 234 ss., disponibile in http://www. penalecontemporaneo.it, ultimo accesso il 24 agosto 2018

37 DOSI, Ettore. La sentenza penale di proscioglimento, Giuffrè, 1955, p. 132. 
classica, il procedimento penale opera come strumento di garanzia dell'imputato in vista dell'accertamento della sua responsabilità per un fatto di cui - sin dalle indagini preliminari e, poi, nella fase processuale - si assume la conformità ad una fattispecie incriminatrice. Sennonché, il lessico del legislatore processuale penale non viene in soccorso a chi voglia assegnare al concetto di fatto un significato univoco ${ }^{38}$. Nella disciplina positiva, il "fatto" è indubbiamente espressione polisemica ${ }^{39}$, perché il relativo concetto viene in gioco in una molteplicità di ambiti non riducibili a unità. Occorre, anzi, rifuggire dalla tentazione di fornire una nozione omnicomprensiva alla locuzione, perché si rischierebbe di aggirare la funzione tipica rivestita dal fatto nei diversi ambiti ${ }^{40}$ : quest'ultimo muta a seconda delle finalità al cui soddisfacimento mira, di volta in volta, l'ordinamento processuale ${ }^{41}$.

Per quanto qui maggiormente preme, è decisiva la necessità d'identificare l'oggetto del giudizio. A dispetto dell'impiego codicistico di espressioni apparentemente sinonimiche (come, ad esempio, "stesso fatto" o "medesimo fatto"), occorre distinguere il relativo significato a seconda che si abbia riguardo all'identificazione del fatto a scopi "endoprocessuali" oppure "extraprocessuali" ${ }^{2}$.

38 Come, del resto, accade anche in ambito sostanziale: cfr., per tutti, FIANDACA, Giovanni. Fatto nel diritto penale, in D. disc. pen., V, Utet, 1991, p. 153.

39 Cfr., in tal senso, FIANDACA, Giovanni. Fatto nel diritto penale, cit., p. 153; GUARNIERI, Giuseppe. Regiudicata (diritto processuale penale), in Noviss. $d$. it., XV, Utet, 1968, p. 231; PAGLIARO, Antonio. Fatto (dir. proc. pen), in Enc. dir., XVI, Giuffrè, 1967, p. 962. In generale, sulla nozione di fatto nel processo penale, cfr., per tutti, RAFARACI, Tommaso. Le nuove contestazioni nel processo penale, cit., passim; UBERTIS, Giulio. Fatto e valore nel sistema probatorio penale, Giuffrè, 1979, passim.

40 Ammoniva dal rischio di «evitare il pericolo di generalizzare [la] nozione di 'fatto'», al fine di applicarla ai differenti ambiti del diritto processuale penale, PAGLIARO, Antonio. Fatto (dir. proc. pen), cit., p. 962. V. anche, di recente, RIVELLO, Pier Paolo. La nozione di "fatto" ai sensi dell'art. 649 c.p.p. e le perduranti incertezze interpretative ricollegabili al principio del ne bis in idem, in Riv. it. dir. proc. pen., 2014, p. 1411 ss.

41 Così, con riguardo ai criteri d'identificazione del fatto alla luce del principio del ne bis in idem, DE LUCA, Giuseppe. Giudicato, Diritto processuale penale, in Enc. giur. Treccani, XV, 1989, p. 10

42 In tal senso v., volendo, CASSIBBA, Fabio. L'imputazione e le sue vicende, in UBERTIS, Giulio; VOENA, Giovanni Paolo (diretto da), Trattato di procedura penale, vol. XXXI, Giuffrè, 2016, p. 22 ss. 
Sul primo versante, viene in gioco l'esigenza d'individuare il fatto in rapporto all'evoluzione dell'accusa nel corso del processo, una volta esercitata l'azione penale: la necessaria premessa è che, se il contenuto dell'accusa è suscettibile di essere descrittivamente modificato, nondimeno il giudizio deve vertere sempre sul medesimo fatto ${ }^{43}$. Dunque, l'identità del fatto trova qui come parametro di valutazione l'enunciato descrittivo dell'imputazione ${ }^{44}$.

Sul secondo versante, vengono in gioco i rapporti fra diversi procedimenti che, in ipotesi, vertono sul medesimo fatto: rileva la litispendenza penale ${ }^{45}$, come accade, per l'appunto, quando si tratti di cogliere la portata del ne bis in idem (art. 649 comma 1 c.p.p.) ${ }^{46}$. Qui,

43 Cfr., fra gli altri, CORDERO, Franco. Considerazioni sul principio d'identità del fatto, in Riv. it. dir. proc. pen., 1958, p. 939 ss.; DOMINIONI, Oreste. L'esercizio dell'azione penale (l'imputazione) (1970), in Le parti nel processo penale. Profili sistematici e problemi, Giuffrè, 1985, p. 134; RAFARACI, Tommaso. Le nuove contestazioni nel processo penale, cit., p. 20 ss. e passim.

In presenza della necessità di procedere a modificare l'imputazione, la verifica circa il permanere dell'identità del fatto a seguito della modifica contenutistica dell'imputazione si esaurisce nel raffronto fra l'enunciato oggetto dell'imputazione originaria e quello oggetto dell'imputazione "modificata". La conclusione non muta quando, nei giudizi d'impugnazione, il giudice è investito del potere di verificare il rispetto del vincolo di correlazione fra l'accusa contestata nell'imputazione e quella effettivamente oggetto della sentenza impugnata. Anche in tal caso, dev'essere assicurata la piena identità fra il fatto contestato nell'imputazione e quello ritenuto in sentenza: posto che il giudizio è operazione decisoria a «tema obbligato» [KOSTORIS, Roberto Ettore. Giudizio (diritto processuale penale), in Enc. giur. Treccani, vol. VI, Roma, 1997, p. 8], il giudice non deve assumere una decisione sul merito dell'accusa che esorbiti dai confini previamente tracciati dal pubblico ministero. Sul tema, con ampiezza, anche per ulteriori riferimenti bibliografici, cfr. BONTEMPELLI, Manfredi. La litispendenza penale, cit., passim.

46 Senza esorbitare dell'oggetto del lavoro, è appena il caso di notare che la stessa conclusione vale quando occorra risolvere il conflitto pratico fra giudicati (art. 669 c.p.p.), oppure ai fini della litispendenza nel caso dell'insorgere di un conflitto di competenza (art. 28 comma 1 c.p.p.), o dell'applicazione della disciplina della retrodatazione dell'efficacia della misura cautelare nel caso delle c.d. contestazioni a catena (art. 297 comma 3 c.p.p.). Analogo discorso, poi, opera in ordine al concetto di «medesimo fatto» ex art. 345 comma 1 c.p.p., la cui individuazione è decisiva ai fini del vaglio circa la riproponibilità dell'azione penale a seguito della sopravvenienza di una condizione di procedibilità, o, ancora, mutatis mutandis, in ordine alla "diversità" del procedimento ai fini della c.d. circolazione delle intercettazioni, la cui utilizzabilità è 
sarebbe irragionevole basare il giudizio d'identità del fatto sulla riscontrata identità contenutistica dell'accusa elevata rispettivamente nelle due vicende giudiziarie. Se la litispendenza fosse risolta sulla base della sovrapponibilità contenutistica delle due accuse, il giudizio sull'identità del fatto nei diversi procedimenti potrebbe non avere mai esito positivo. Il "fatto" sarebbe sempre descrivibile in modo "diverso": una "variante qualunque della seconda domanda $\gg{ }^{47}$ basterebbe ad escludere la medesimezza del fatto, a tutto discapito delle esigenze di garanzia e di certezza delle situazioni giuridiche. Se così è, il criterio volto a verificare l'identità del fatto si basa su una nozione più ampia, che prescinde dallo stretto contenuto dell'imputazione. Il giudizio d'identità riposa sulla descrizione della condotta e dell'eventuale evento naturalistico, individuato alla luce dell'oggetto materiale su cui essa ricade (condotta c.d. transitiva) ${ }^{48}$. In questo senso, la nozione di fatto processualmente rilevante coincide con quella accolta nell'ambito della c.d. teoria bipartita del reato ${ }^{49}$, essendo irrilevanti, invece, i profili attinenti alla colpevolezza.

Più precisamente, ai fini del ne bis in idem si è in presenza del medesimo fatto quando la variazione descrittiva dell'episodio non investa gli appena rammentati elementi nucleari dell'episodio oggetto della sentenza passata in giudicato ${ }^{50}$. Dunque, ogni variazione delle

regolata dall'art. 270 c.p.p. in chiave derogatoria e restrittiva rispetto al regime ordinario di ammissibilità.

47 Così, circa la portata dell'effetto preclusivo sprigionato dal giudicato su un certo fatto, CORDERO, Franco. Procedura penale, cit., p. 1206.

48 Così, ancora, CORDERO, Franco. Considerazioni sul principio d'identità del fatto, cit., spec. p. 939 ss. V. anche CAPRIOLI, Francesco. Il principio del ne bis in idem, cit., p. 85 ss.; CORDERO, Franco. Procedura penale, cit., p. 1206.

49 Cfr. ANTOLISEI, Francesco. Manuale di diritto penale. Parte generale, XIV ed. aggiornata e integrata da L. Conti, Giuffrè, 1997, p. 215 ss.; MANTOVANI, Ferrando. Diritto penale, Cedam, 2017, p. 102.

50 In tal senso si orienta l'indirizzo largamente prevalente: cfr. CAPRIOLI, Francesco. Il principio del ne bis in idem, cit., p. 85 ss.; CORDERO, Franco. Procedura penale, cit., p. 1207 ss.; PAGLIARO, Antonio. Fatto (dir. proc. pen), in Enc. dir., XVI, Giuffrè, 1967, p. 964; RAFARACI, Tommaso. Ne bis in idem, cit., p. 872. Sul tema, v. pure CALLARI, Francesco. La firmitas del giudicato penale: essenza e limiti, Milano, 2009, spec. p. 141 ss.; MANCUSO, Enrico Maria. Il giudicato nel processo penale, cit., p. 451 ss.; DANIELLE, Marcello. Profili sistematici della sentenza di non luogo a procedere, Torino, 2005, p. 178179; QUATTROCOLLO, Serena. Riqualificazione del fatto nella sentenza penale 
modalità della condotta ${ }^{51}$, dell'evento naturalistico (purché ricada sul medesimo bene giuridico $)^{52}$, del nesso di causalità, oltre che dell'elemento soggettivo ${ }^{53}$, non incide sull'identità del fatto e resta coperta dall'effetto preclusivo del giudicato.

Quanto alla variazione delle coordinate spaziali e temporali della condotta, il giudizio sull'identità del fatto ai fini del ne bis in idem implica, però, un'ulteriore verifica. Occorre accertare se le condotte - l'una, già considerata nella sentenza passata in giudicato, l'altra, oggetto del nuovo esercizio dell'azione penale - siano, astrattamente, non reiterabili nel mondo fisico. In tal caso, posto che nel mondo fisico i fatti accadono una

e tutela del contraddittorio, Jovene, 2011, p. 94 ss. In giurisprudenza, Cass., sez. un., 28 giugno 2005, Donati ed altro, cit., p. 28 ss. Nel senso, invece, che il ne bis in idem processuale va escluso in presenza di «fattispecie giudiziali diverse», cfr. già LOZZI, Gilberto. Profili di una indagine sui rapporti tra «ne bis in idem» e concorso formale di reati, Milano, 1974, spec. p. 57 ss.; cfr., altresì, RIVELLO, Pier Paolo. Analisi in tema di ne bis in idem, in Riv. it. dir. proc. pen., 1991, p. 500 ss.; Id., La nozione di "fatto" ai sensi dell'art. 649 c.p.p.e le perduranti incertezze interpretative ricollegabili al principio del ne bis in idem, ivi, 2014, p. 1422 ss.

51 Si pensi, ad esempio, all'uccisione d'un individuo procurata con l'impiego di un coltello o di una bottiglia di vetro spezzata; o, ancora, alle lesioni dolose procurate con un'aggressione avvenuta frontalmente o di spalle; o, infine, alla truffa perpetrata esibendo un falso tesserino di riconoscimento o un falso contratto di mandato. Ancora, in forza dell'art. 649 comma 1 c.p.p., resta irrilevante, ai fini del mutamento del fatto, che vengano contestate circostanze aggravanti.

52 Viene in gioco la c.d. progressione criminosa o la variazione di «grado», secondo quando previsto dall'art. 649 comma 1 c.p.p. Si allude, più precisamente, alle ipotesi in cui il pubblico ministero, passata in giudicato la sentenza per il delitto nella forma tentata, pretenda, poi, di contestare lo stesso reato nella forma consumata. Analoga conclusione vale, poi, con riguardo al passaggio da un grado dell'offesa ad uno più intenso, come accade per le ipotesi in cui, passata in giudicato la sentenza per il delitto di lesioni ai danni di $\mathrm{X}$, il pubblico ministero pretenda di procedere per il delitto di omicidio nei confronti della medesima persona offesa. In dottrina, CAPRIOLI, Francesco. Il principio del ne bis in idem, cit., p. 85 ss.; CORDERO, Franco. Procedura penale, cit., p. 1210-1211; MANCUSO, Enrico Maria. Il giudicato nel processo penale, cit., p. 466 ss.

53 Neppure quando la variazione comportasse una diversa qualificazione del fatto (quando si passasse, ad esempio, dall'ipotesi colposa a quella dolosa). L'art. 649 comma 1 c.p.p. rende espressamente irrilevanti le variazioni che investano il «titolo» del reato. 
volta sola ${ }^{54}$, oggetto del nuovo esercizio dell'azione penale sarà lo stesso fatto già considerato dal giudicato, scattando, così, l'effetto preclusivo dell'art. 649 c.p.p. Se, invece, la condotta sia reiterabile, la variazione delle coordinate spaziali e temporali pone il dubbio se essa integri una diversa descrizione del medesimo fatto (dunque, coperto dall'effetto preclusivo) oppure, direttamente, la descrizione di un altro fatto (dunque, non coperto dall'effetto preclusivo) ${ }^{55}$. Si rientra nella nozione codicistica di

54 Così, CORDERO, Franco. Procedura penale, cit., p. 455-456. V. pure RIVELLO, Pier Paolo. La nozione di "fatto" ai sensi dell'art. 649 c.p.p. e le perduranti incertezze interpretative ricollegabili al principio del ne bis in idem, cit., p. 1431 ss.

55 Si pensi all'ipotesi in cui, descritta la rapina presso la Banca Z come avvenuta il giorno $x$, si contesti, poi, che l'episodio sia avvenuto il giorno $y$.

Ancora diverso è il rilievo delle coordinate spaziali e temporali in rapporto alla figura del reato permanente e di quello abituale: per l'inquadramento teorico del problema, cfr., per tutti, CAPRIOLI, Francesco. Il principio del ne bis in idem, cit., p. 99-100; MANCUSO, Enrico Maria. Il giudicato nel processo penale, cit., p. 477 ss. Tutto dipende dalla legittimazione data alla c.d. contestazione cronologicamente aperta dei fatti di cui all'imputazione. Ove si riconosca la legittimità della sola contestazione "chiusa", relativa cioè a condotte puntualmente descritte e cronologicamente delimitate da un momento iniziale e da un momento finale, resta coperto dal giudicato tutto ciò che è espressamente contestato nell'accusa (cfr. CORDERO, Franco. Procedura penale, cit., p. 1212). Così, il ne bis in idem avrebbe, a sua volta, una portata oggettiva precisamente delimitata: l'effetto preclusivo scatterebbe in ordine alle condotte già considerate entro quel preciso lasso temporale. La giurisprudenza riconosce, invece, che il pubblico ministero possa non circoscrivere la contestazione entro precisi confini temporali, così lasciando aperto il momento di cessazione della permanenza o della abitualità della condotta. Da qui, una duplice conseguenza: per un verso, la cessazione della permanenza viene fatta coincidere con la data di pronuncia della sentenza di primo grado (Cfr., fra le altre, Cass., sez. V, 19 marzo 2009, Marazia, in CED, n. 244479; Cass., sez. V, $1^{\circ}$ luglio 1996, Aiello, ivi, n. 206292); per l'altro, in caso di incertezza circa il momento di effettiva cessazione della permanenza, tocca al giudice, anche dopo il passaggio in giudicato della sentenza, procedere al relativo accertamento (Cfr. Cass., sez. I, 24 ottobre 2013, Formicola, in CED, n. 257725). E' evidente, però, che così opinando, si lascia nell'incertezza l'individuazione del fatto, in ordine non solo all'oggetto del giudizio, ma anche in rapporto alla portata oggettiva dell'effetto preclusivo della relativa sentenza una volta passata in giudicato. Cfr. CAPRIOLI, Francesco. Il principio del ne bis in idem, cit., p. 100. D'altra parte, non soddisfa neppure il tentativo operato da parte della dottrina di far coincidere col momento di passaggio in giudicato della sentenza in parola i confini del ne bis in idem: cfr. LOZZI, Gilberto. Lezioni di procedura penale, Giappichelli, 2016, p. 813; v. anche BENE, Teresa. 
fatto nuovo, non coperto dal giudicato, ogni qualvolta sia possibile predicare la contestuale verità dei due enunciati, ove la condotta compare diversamente orientata nello spazio e nel tempo.

Dalla ricostruzione proposta emerge che la modifica endoprocessuale dell'imputazione e l'effetto preclusivo del giudicato sull'idem factum occupano spazi complementari ${ }^{56}$. In breve, il concetto di idem factum rilevante ai fini della garanzia del ne bis in idem non può essere impiegato come criterio per risolvere la questione dell'identità del fatto in rapporto alle vicende modificative dell'imputazione e dell'effettivo rispetto del vincolo di correlazione fra accusa e sentenza ${ }^{57}$.

L'analisi relativa ai presupposti operativi del ne bis in idem resterebbe incompleta se non si considerasse almeno un'ipotesi in cui, sin dal piano normativo, la mera individuazione del fatto coperto dal giudicato non è sufficiente per individuare, specularmente, la portata dell'effetto preclusivo della sentenza. Talvolta, l'operatività del divieto di secondo giudizio «dipende dal contenuto della sentenza divenuta irrevocabile», come nell'ipotesi di reati previsti da fattispecie alternative, perché chi sia stato processato per una delle condotte criminose alternative può essere giudicato per un'altra solo se è stato assolto; non, invece, se è stato condannato ${ }^{58}$.

Reato permanente e modifica dell'imputazione, cit., c. 647; CALAMANDREI, Piero. Diversità del fatto e modifica dell'imputazione nel codice di procedura penale del 1988, in Riv. it. dir. proc. pen., 1996, p. 653-654. La soluzione, pur apprezzabilmente volta a dare certezza alle situazioni giuridiche soggettive, non esclude la riprosecution per la stessa condotta, quando l'azione penale sia stata nuovamente esercitata anteriormente al passaggio in giudicato della prima sentenza, con l'inevitabile dispendio di risorse.

Così, CORDERO, Franco. Procedura penale, cit., p. 1206-1207; LOZZI, Gilberto. Lezioni di procedura penale, cit., p. 807.

Cfr. CAPRIOLI, Francesco. Il principio del ne bis in idem, cit., p. 96 ss.; MANCUSO, Enrico Maria. Il giudicato nel processo penale, cit., p. 445 ss.; QUATTROCOLO, Serena. Riqualificazione del fatto nella sentenza penale e tutela del contraddittorio, cit., p. 94 ss.; RAFARACI, Tommaso. Le nuove contestazioni nel processo penale, cit., spec. p. 13 ss.

In tali precisi termini, CAPRIOLI, Francesco. Il principio del ne bis in idem, cit., p. 100, che evidenzia come sia la legge sostanziale, non quella processuale, ad impedire di sanzionare due volte la stessa persona per condotte alternative, benché i fatti siano diversi (ivi, p. 101). In breve, si tratta di un 


\subsection{LE PRASSI ELUSIVE DEL NE BIS IN IDEM NELLA GIURISPRUDENZA NAZIONALE E LA REAZIONE DELLA CoRTE COSTITUZIONALE}

Una considerazione autonoma merita il complesso rapporto fra la figura del concorso formale di reati e la portata del ne bis in idem ${ }^{59}$.

Qui, l'individuazione dell'effetto preclusivo sprigionato dal giudicato passa, anzitutto, attraverso la necessità di discriminare il concorso formale fra reati di pura condotta o ad evento naturalistico. Per questi ultimi, posto che l'idem factum è rappresento dalla condotta e dall'oggetto materiale su cui essa ricade ${ }^{60}$, si è in presenza di tanti fatti, fra loro autonomi, quanti sono gli oggetti materiali su cui cade l'unica condotta. L'ipotesi più semplice è che la condotta ricada su oggetti fisici diversi. Si pensi all'unico colpo di arma da fuoco sparato da Tizio che colpisca Caio, Mevio e Sempronio, rispettivamente uccidendo il primo e ferendo i secondi: nessun dubbio, il passaggio in giudicato per il delitto di omicidio doloso di Caio, non esclude la riprosecution per gli episodi di lesioni dolose e viceversa. Tuttavia, un'unica condotta transitiva può cadere sullo stesso oggetto nel mondo fisico e generare una pluralità di autonomi eventi naturalistici: anche in tal caso, il giudicato relativo ad uno degli eventi non vieta l'autonomo giudizio sugli altri ${ }^{61}$. Ancora, il divieto di secondo giudizio non opera neppure quando l'unica condotta abbia violato diverse fattispecie incriminatrici, una sola delle quali integri un reato ad evento naturalistico. Si pensi alla condotta di chi, dal proprio balcone di casa, abbia sparato un colpo di arma da fuoco in aria, allo scopo di festeggiare la vittoria della propria nazionale ai mondiali di calcio, provocando, però, la morte di un passante. Il passaggio in giudicato della sentenza relativa

tipico caso di ne bis in idem penale: così, CORDERO, Franco. Procedura penale, cit., p. 1212-1213.

59 Sul tema, assai complesso, v. con ampiezza, per tutti, anche per ulteriori riferimenti, CAPRIOLI, Francesco. Il principio del ne bis in idem, cit., p. 87 ss.; CORDERO, Franco. Procedura penale, cit., p. 1206 ss.; MANCUSO, Enrico Maria. Il giudicato nel processo penale, cit., p. 451 ss.; da ultimo, GALANTINI, Novella. Il 'fatto' nella prospettiva del divieto di secondo giudizio, cit., p. 1209 ss. V. supra, § 2.2 .

61 Cfr. CAPRIOLI, Francesco. Il principio del ne bis in idem, cit., p. 93, che allude alla condotta - non coperta dal ne bis in idem - di chi, resistendo a un pubblico ufficiale, gli procuri lesioni personali. 
al disturbo alla quiete pubblica (reato di pura condotta) non esclude il giudizio per l'omicidio del passante (reato ad evento naturalistico).

Diverse sono le conclusioni per l'ipotesi del concorso formale fra reati di pura condotta. Difettando, per definizione, l'evento naturalistico, ai fini del ne bis in idem il fatto coincide qui con la condotta ${ }^{62}$. Così, o tutte le violazioni sono cumulativamente contestate dal pubblico ministero nel medesimo processo oppure il passaggio in giudicato della sentenza relativa ad una violazione sprigiona l'effetto preclusivo per le altre ${ }^{63}$, restando irrilevante che vengano, poi, contestati dal pubblico ministero un nomen iuris e la lesione ad un bene giuridico diversi da quelli già considerati nella sentenza passata in giudicato ${ }^{64}$.

La ricostruzione si allinea con l'inequivoca formula dell'art. 649 comma 1 c.p.p., in forza della quale la diversa qualificazione giuridica («titolo») data al fatto non ne muta l'identità e, dunque, resta irrilevante ai fini del divieto di secondo giudizio. Peraltro, proprio a dispetto di quella formula, la giurisprudenza della Corte di cassazione ha legittimato un costante aggiramento della garanzia prevista dall'art. 649 c.p.p., escludendo che il concorso formale di reati, anche quando essi siano di pura condotta, faccia scattare il ne bis in idem: il pubblico ministero sarebbe sempre legittimato a procedere per un reato in concorso formale con un altro fatto, su cui sia già sceso il giudicato, salva l'ipotesi in cui il giudicato assolutorio fosse stato pronunciato perché il fatto non sussiste o perché l'imputato non lo ha commesso ${ }^{65}$.

$\mathrm{Al}$ di là del già evidenziato contrasto con la lettera dell'art. 649 comma 1 c.p.p. e dell'irragionevole (e illogica) distinzione praticata fra

62 Si pensi al caso del concorso formale eterogeneo fra il delitto di falsa testimonianza e quello di calunnia: cfr. CAPRIOLI, Francesco. Il principio del ne bis in idem, cit., p. 92; LOZZI, Gilberto. Lezioni di procedura penale, cit., p. 810.

63 Così, CAPRIOLI, Francesco. Il principio del ne bis in idem, cit., p. 95, si rendono «non più rimediabili ... gli errori strategici commessi dal pubblico ministero», che non avesse proceduto a contestare tutte le ipotesi di reato fra loro concorrenti nel corso del primo processo poi definitivo con sentenza irrevocabile.

64 Così, MANCUSO, Enrico Maria. Il giudicato nel processo penale, cit., p. 453.

65 Così, fra le altre, Cass., sez. III, 15 aprile 2009, Ferrarelli, in Giur. it., 2010, p. 1181 ss., con motivazione e con nota critica di CAPRIOLI, Francesco; Cass., sez. VI, 9 ottobre 2007, Nocchiero, in CED, n. 238442; Cass., sez. I, 18 maggio 2004, Purpura, in Cass. pen., 2006, p. 183, m. 56. 
gli effetti del giudicato di condanna e quelli del giudicato di assoluzione "con formula piena" ${ }^{66}$, qui preme maggiormente evidenziare un autonomo profilo critico. La conclusione propugnata dalla Corte di cassazione si pone in frizione con l'obiettivo di assicurare una tutela effettiva al soggetto già irrevocabilmente prosciolto o condannato per il medesimo fatto, posta al centro delle argomentazioni spese in materia dalla Corte di Giustizia e dalla Corte europea ${ }^{67}$.

Il rilievo è decisivo per comprendere perché la Corte costituzionale abbia - assai di recente - represso l'indirizzo della Corte di cassazione, dichiarando l'illegittimità costituzionale dell'art. 649 comma 1 c.p.p., per un contrasto con l'art. 117 comma 1 Cost., in relazione all'art. 4 prot. n. 7 CEDU, «nella parte in cui esclude che il fatto sia il medesimo per la sola circostanza che sussiste un concorso formale tra il reato già giudicato con sentenza divenuta irrevocabile e il reato per cui è iniziato il nuovo procedimento penale ${ }^{68}$. Emergono prepotentemente gli effetti

66 Secondo la Cassazione, dati i reati X e Y in concorso formale, condannato irrevocabilmente l'imputato da X, non resterebbe preclusa l'azione penale $\mathrm{Y}$, essendo Y diverso da X; tuttavia, assolto irrevocabilmente l'imputato da X, l'azione penale sarebbe invece preclusa su Y, sul presupposto che Y è caratterizzato dalla medesima condotta di $X$. Talché, delle due l'una: o i due fatti non sono mai diversi, in forza della medesima condotta, e allora il ne bis in idem sarebbe destinato ad operare sempre, indipendentemente dall'esito del primo processo; oppure i fatti sono sempre diversi e allora il ne bis in idem sarebbe destinato a non operare mai. Così, CAPRIOLI, Francesco. Sui rapporti fra ne bis in idem processuale e concorso di reati, in Giur. it., 2010, p. 1186 ss.

67 Sul tema, per tutti, GALANTINI, Novella. Il 'fatto' nella prospettiva del divieto di secondo giudizio, cit., p. 1209 ss.

68 Così, C. cost., sent. 21 luglio 2016 n. 200, cit., p. 60 ss., sulla cui portata, con vari accenti, FERRUA, Paolo. La sentenza costituzionale sul caso Eternit: il ne bis in idem tra diritto vigente e diritto vivente, cit., p. 78 ss.; LAVARINI, Barbara. Il 'fatto' ai fini del ne bis in idem nella legge italiana e nella CEDU: la Corte costituzionale alla ricerca di un difficile equilibrio, ci., p. 60 ss.; GALANTINI, Novella. Postilla ad uno scritto in tema di ne bis in idem, cit., p. 3 ss.; PULITANÒ, Domenico. La Corte costituzionale sul ne bis in idem, in Cass. pen., 2017, p. 70 ss.; Id., Ne bis in idem. Novità dalla Corte e problemi aperti, in Dir. pen. proc., 2016, p. 1588 ss.; RIVELLO, Pier Paolo. I rapporti tra giudizio penale e tributario ed il rispetto del principio del ne bis in idem, cit., p. 118 ss. La pronuncia è tanto più significativa perché, in un lontano precedente, la Corte costituzionale aveva già dichiarato infondata un'analoga questione, sollevata a proposito della portata dell'art. 90 c.p.p. 1930 in rapporto al concorso formale di reati: cfr. C. cost., sent. 15 gennaio 1976 n. 6, in Giur. cost., 1976, p. 33. 
positivi della "tutela multilivello" dei diritti fondamentali ${ }^{69}$, fondata su una reciproca interrelazione, non solo fra la fonte nazionale e quella sovranazionale, ma anche fra la giurisprudenza interna e l'elaborazione dei giudici sovranazionali, con l'obiettivo di pervenire interpretazioni "virtuose" che assicurino la «massima espansione delle garanzie» ${ }^{70}$.

I giudici di Palazzo della Consulta ricostruiscono la portata dell'art. 649 comma 1 c.p.p. muovendo dall'art. 4 prot. 7 CEDU e, più in particolare, dalla premessa secondo cui è ormai «pacifico ... che la Convenzione recepisce il più favorevole criterio dell'idem factum, a dispetto della lettera dell'art. 4 del Protocollo n. 7, anziché la più restrittiva nozione di idem legale» ${ }^{71}$. Quanto all'identificazione del fatto, poi, in prospettiva sistematica (e valorizzando lo scopo del ne bis in idem), non v'è alcuna ragione per cui rilevi la sola condotta e non anche l'evento naturalistico, ossia la modificazione della realtà che è conseguita alla condotta; anzi, anche la consolidata giurisprudenza della Corte di Strasburgo milita in tal senso. Inoltre, il principio del ne bis in idem ricopre un ruolo centrale nell'ordinamento in funzione di garanzia, come principio di civiltà, volto anche a tutelare l'inviolabilità della libertà personale e la certezza delle situazioni giuridiche. Da qui, una duplice conseguenza: da un lato, le disposizioni codicistiche non possono che essere lette alla luce dei primari valori costituzionali in gioco; dall'altro, non suonano compatibili con la protezione degli interessi costituzionali in gioco prassi volte ad aggirare la ratio di garanzia dell'art 649 c.p.p. Se così è, «[s]ulla base della triade condotta-nesso causale-evento naturalistico, il giudice può affermare che il fatto oggetto del nuovo giudizio è il medesimo solo se riscontra la coincidenza di tutti questi elementi, assunti in una dimensione empirica», essendo irrilevante - in presenza della riscontrata medesimezza di tali elementi - la qualificazione giuridica del reato e la sussistenza di un'ipotesi di concorso formale fra reati ${ }^{72}$.

69 V. supra, § 1.

70 Cui deve sempre tendere «il confronto tra tutela convenzionale e tutela costituzionale dei diritti fondamentali» (C. cost., sent. 4 dicembre 2009 n. 317, in Giur. cost., 2009, p. 4761).

71 C. cost., sent. 21 luglio 2016 n. 200, cit., p. 63, da cui è tratta anche l'argomentazione che immediatamente segue nel testo.

72 Così, ancora, C. cost., sent. 21 luglio 2016 n. 200, cit., p. 69. 
La ricostruzione della Corte costituzionale - dichiarando costituzionalmente illegittimo l'art. 649 comma 1 c.p.p. nel significato datogli dal «diritto vivente» - ha inteso evitare che si potesse perpetuare un indirizzo della Corte di Cassazione in contrasto sia con la lettera della previsione in parola sia con la consolidata interpretazione dell'art. 4 prot. 7 CEDU da parte della Corte europea $^{73}$. Resta, così, confermato il circolo virtuoso generato in materia dalla tutela multilivello dei diritti fondamentali, ove il «dialogo», talvolta teso ${ }^{74}$, fra le Corti sovranazionali e i giudici comuni si risolve in un'espansione delle garanzie.

\section{LA "NUOVA FRONTIERA": NE BIS IN IDEM E DOPPIO BINARIO SANZIONATORIO}

Pur seguendo percorsi esegetici a tratti tortuosi, la giurisprudenza, sovranazionale e nazionale, è pervenuta ad un'univoca nozione di idem factum rilevante in ordine alla portata oggettiva del ne bis in idem. Sotto questo profilo, l'ordinamento nazionale pare aver raggiunto un punto di equilibrio: la stabilizzazione del sistema attorno ad un univoco concetto di fatto si riverbera positivamente sull'effettivo rispetto del canone della legalità che, fra le sue molteplici implicazioni, annovera l'esigenza di una stretta osservanza del principio di eguaglianza.

Sennonché, l'effettivo rispetto del canone in parola in materia di ne bis in idem è, oggi, ancora condizionato dall'incertezza che regna in

73 Cfr., in tal senso, FERRUA, Paolo. La sentenza costituzionale sul caso Eternit: il ne bis in idem tra diritto vigente e diritto vivente, cit., p. 82. V. anche, anteriormente alla sent. cost. n. 200 del 2016, GALANTINI, Novella. Il 'fatto' nella prospettiva del divieto di secondo giudizio, cit., p. 1214.

74 Senza addentrarsi in un argomento extra-vagante ai fini del presente lavoro, si pensi all'acceso dibattito in relazione ai «controlimiti» costituzionali che la Corte costituzionale ha ritenuto di poter invocare per impedire che l'adeguamento a pronunce della Corte di Giustizia vada a tutto discapito dei principi supremi dell'ordinamento: cfr. C. cost., sent. 26 gennaio 2017 n. 24, in Giur. cost., 2017, p. 171 ss., con osservazioni di Celotto ed Esposito; sul tema venuto alla ribalta in tempi davvero recenti - la letteratura è già molto ampia: cfr., per una silloge delle posizioni in materia, i contributi multidisciplinari raccolti in BERNARDI, Alessandro (a cura di), I controlimiti. Primato delle norme europee e difesa dei principi costituzionali, Jovene, 2017; BERNARDI, Alessandro; CUPELLI, Cristiano (a cura di), Il caso Taricco e il dialogo tra le Corti. L'ordinanza 24/2017 della Corte costituzionale, Jovene, 2017. 
ordine ai limiti del ne bis in idem quando lo stesso fatto sia assoggettato dall'ordinamento nazionale ad un "doppio binario sanzionatorio"

La ragione prima dell'incertezza deriva dalla circostanza che la nozione di «ambito penale» è formalmente delimitata dalla legge sul piano nazionale mentre ne è sostanzialmente svincolata sul piano sovranazionale. In estrema sintesi, le fonti sovranazionali si caratterizzano per lo scopo di scongiurare la "truffa delle etichette", in aperta elusione delle garanzie penalistiche: per consolidata giurisprudenza della Corte europea di Strasburgo e della Corte di giustizia del Lussemburgo, non è sufficiente che l'ordinamento nazionale qualifichi una sanzione come «non penale» per legittimarne una applicazione non rispettosa delle garanzie sostanziali e processuali previste dalla disciplina sovranazionale proprie della matière pénale $^{75}$. Piuttosto, affinché una sanzione abbia natura sostanzialmente penale occorre avere riguardo: a) alla sua formale qualificazione in tal senso da parte della legge; b) alla sua gravità; c) alla sua finalità afflittiva. Ne deriva che una sanzione resta comunque attratta entro la matière pénale quando - pur qualificata come amministrativa dalla sua fonte regolatrice nazionale - appaia sostanzialmente avere natura penale in ragione della gravità dei suoi effetti nella sfera giuridica di chi la subisca e delle finalità afflittive conseguite dall'ordinamento con la sua irrogazione.

\subsection{NE BIS IN IDEM E DOPPIO BINARIO SANZIONATORIO NELLE FONTI SOVRANAZIONALI}

Si comprende, così, perché la giurisprudenza della Corte di giustizia, prima ${ }^{76}$, e della Corte europea, in seguito ${ }^{77}$, si siano attestate su

75 Sul primo versante, si pensi al canone nulla poena sine lege, proclamato dall'art. 7 Conv. eur. dir. uomo, con i sui corollari, fra l'altro, della necessaria determinatezza delle fattispecie incriminatrici e dell'irretroattività della norma più sfavorevole; sul secondo, si pensi, fra le altre, alle garanzie previste in favore dell'accusato tipiche del fair trail in ambito penale, proclamate dall'art. 6 comma 3 Conv. eur. dir. uomo e, per l'appunto, di quella ne bis in idem, riconosciuto dall'art. 4 prot. n. 7 CEDU.

76 Cfr., fra le altre, C. giust. UE, sez. II, 9 marzo 2006, C-436/04, Léopold Henri Van Esbroek; C. giust. UE, sez. II, 28 settembre 2006, C-150/05, Jan Leo Van Straaten

77 Corte e.d.u., grande camera, 10 febbraio 2009, Zolotukhin c. Russia, i cui argomenti sono stati, in seguito, ampiamente ripresi da Corte e.d.u., sez. II, 4 marzo 2014, Grande Stevens e altri c. Italia, spec. § 219 ss. 
una nozione non formale del concetto di decisione irrevocabile, volta ad escludere che il medesimo fatto possa essere sanzionato due volte da parte delle autorità nazionali in due "procedimenti paralleli» ${ }^{78}$. In effetti, dare rilievo ad una decisione "sostanzialmente penale" ai fini del divieto di secondo giudizio schiude immediatamente la via alla necessità di verificare se e come operi la garanzia in parola in rapporto a una pluralità di procedimenti svolti in contesti diversi ${ }^{79}$.

Per la Corte di Strasburgo, la pluralità di procedimenti rileva in una dimensione sincronica: la garanzia del ne bis in idem non vieta la litispendenza fra procedimenti, tutti, o solo alcuni, aventi natura formalmente penale ${ }^{80}$; la garanzia scatta, non già per impedire il contestuale svolgimento di procedimenti, ma per impedire la duplicazione di sanzioni, sul presupposto che una decisione sia diventata definitiva.

Ciò posto, per lungo tempo, la Corte europea aveva subordinato l'operatività del ne bis in idem in materia di procedimenti paralleli alla triplice condizione che: $a$ ) i due procedimenti (nella sede penale e in quella extrapenale) fossero stati promossi nei confronti della stessa persona e avessero ad oggetto la stessa condotta $^{81} ; b$ ) in uno dei due procedimenti fosse stata pronunciata una decisione definitiva; $c$ ) il pro-

78 Secondo la dizione che compare in Corte e.d.u., grande camera, 15 novembre 2016, A e B c. Norvegia, §§ 122-123 e 130. Tocca appena notare che l'espressione «procedimenti paralleli» qui impiegata dalla Corte europea non coincide con quella di cui all'art. 3 decisione quadro 2009/948/GAI, relativa al divieto di secondo giudizio con riguardo a procedimenti penali svolti in diversi Stati dell'Unione europea: cfr. BUZZELLI, Silvia. Procedimenti paralleli, spazio di giustizia, Unione europea: il contesto normativo e gli aspetti problematici, in Arch. pen., 2012, n. 1, p. 1 ss.

79 Benché la Corte europea evidenzi come, in linea di principio, il divieto di bis in idem sarebbe assicurato da un sistema in cui il medesimo fatto venisse punito con diverse sanzioni in un unico procedimento: così, Corte e.d.u., grande camera, 15 novembre 2016, A e B c. Norvegia; v. anche Corte e.d.u., sez. I, 30 aprile 2015, Kapetanios c. Grecia.

80 Cfr., anzitutto, C. eur dir. uomo, grande camera, 10 febbraio 2009, Zolotukhin c. Russia, § 107; nonché, fra le altre, sez. V, 16 giugno 2016, Igor Tarasov c. Ucraina, § 31; sez. V, 27 novembre 2014, Lucky Dev c. Svezia, § 60. In dottrina, anche per ulteriori riferimenti, BONTEMPELLI, Manfredi. La litispendenza penale, cit., p. 64 ss.

81 Cfr., per tutte, C. eur. dir. uomo, sez. II, 4 marzo 2014, Grande Stevens e altri c. Italia, § 224 e 227. 
cedimento extrapenale fosse da considerare, in effetti, matière pénale, secondo gli appena rammentati "criteri Engel" 2 .

Più di recente, la Corte europea ha mutato sensibilmente indirizzo: dopo la sentenza resa dalla grande camera nel caso A e B c. Norvegia del 15 novembre $2016^{83}$, prontamente recepita dalle sezioni semplici della medesima Corte ${ }^{84}$, quei tre presupposti sono stati fortemente ridimensionati.

La Corte di Strasburgo - svincolandosi dal testo dell'art. 4 prot. n. 7 CEDU - ha introdotto un nuovo requisito, destinato a prevalere sui primi. La novità sta tutta nella considerazione assegnata alla «sufficiente connessione sostanziale e temporale» fra procedimenti, come criterio decisivo per sciogliere il dubbio sulla violazione dell'art. 4 prot. n. 7 CEDU : in chiave teleologica, il doppio binario sanzionatorio garantisce un «approccio integrato [e] un tutt'uno coerente» nella repressione degli illeciti, perché idoneo a soddisfare interessi diversi, col solo limite

82 Cfr., fra le molte, C. eur. dir. uomo, grande camera, 10 febbraio 2009, Zolotukhin c. Russia; nonché, sez. IV, 13 giugno 2017, Šimkus c. Lituania; sez. I, 18 maggio 2017, Jóhannesson e altri c. Islanda; sez. I, 30 aprile 2015, Kapetanios e altri c. Grecia; sez. II, 27 novembre 2014, Lucky Dev c. Svezia; sez. IV, 20 maggio 2014, Pirttimäki c. Finlandia; sez. II, 4 marzo 2014, Grande Stevens e altri c. Italia.

83 Cfr. Corte e.d.u., grande camera, 15 novembre 2016, A e B c. Norvegia, sulla quale, fra gli altri e da vari punti di vista, oltre agli autori indicati nel prosieguo del lavoro, BRANCACCIO, Matilde, FIDELBO, Giorgio. Ne bis in idem: percorsi giurisprudenziali e recenti approdi della giurisprudenza nazionale ed europea, cit., p. 13 ss.; DE AMICIS, Gaetano; GAETA, Piero. Il confine di sabbia: la Corte Edu ancora di fronte al divieto del ne bis in idem, in Cass. pen., 2017, p. 469 ss.; FIMIANI, Paolo. Market abuse e doppio binario sanzionatorio dopo la sentenza della Corte E.D.U., Grande camera, 15 novembre 2016, A e B c. Norvegia, in Dir. pen. cont., ed. on-line, 8 febbraio 2017, p. 1 ss., disponibile in http://www.penalecontemporaneo.it, ultimo accesso il 24 agosto 2018; MANACORDA, Stefano. Equazioni complesse: il ne bis in idem "ancipite" sul "doppio binario" per gli abusi di mercato al vaglio della giurisprudenza, cit., p. 520 ss.; VIGANOे, Francesco. La Grande camera della Corte di Strasburgo su ne bis in idem e doppio binario sanzionatorio, in Dir. pen. cont., ed. on-line, 18 novembre 2016, disponibile in http://www.penalecontemporaneo.it, ultimo accesso il 24 agosto 2018.

Cfr. C. eur dir. uomo, sez. IV, 13 giugno 2017, Šimkus c. Lituania, § 41 ss.; sez. I, 18 maggio 2017, Jóhannesson e altri c. Islanda, spec. § 49 ss., sulla quale, VIGANÒ, Francesco. Una nuova sentenza di Strasburgo su ne bis in idem e reati tributari, in Dir. pen. cont., ed. on-line, 22 maggio 2017, disponibile in http:// www.penalecontemporaneo.it, ultimo accesso il 24 agosto 2018. 
che la duplicazione delle risposte sanzionatorie non comporti situazioni d'ingiustizia per i cittadini ${ }^{85}$.

Forse consapevoli che il nuovo requisito ampiamente creativo e connotato da scarsa determinatezza, i giudici di Strasburgo si sono prodigati nel tentativo di ancorarne l'accertamento a criteri puntuali ${ }^{86}$. Anzitutto, la sufficiente connessione fra procedimenti deve sussistere, al contempo, sul versante sostanziale e su quello temporale. Sul primo, le «finalità perseguite e gli strumenti impiegati» nei due procedimenti devono essere «complementari»; $i$ «potenziali effetti» derivanti dai procedimenti paralleli, a loro volta, devono essere «proporzionati e prevedibili dagli individui coinvolti» ${ }^{87}$. Sul secondo, i due procedimenti non devono indefettibilmente svolgersi in maniera simultanea dall'inizio alla fine; piuttosto, la contiguità temporale dev'essere «sufficientemente stretta da proteggere gli individui dal rischio di essere sottoposti a incertezza e ritardi derivanti dalla [loro] protrazione».

$\mathrm{Al}$ di là delle considerazioni di politica criminale (che, per vero, dovrebbero essere estranee agli apprezzamenti della Corte di Strasburgo), risolte nell'ampia legittimazione tributata dalla grande camera al doppio binario sanzionatorio ${ }^{88}$, la pronuncia della Corte europea espone

85 Cfr. C. eur dir. uomo, grande camera, 15 novembre 2016, A e B c. Norvegia, $\S \S 122-123$ e 130.

86 In effetti, ancorché in un sistema in cui il case-law riveste un ruolo centrale, la legalità impone alla Corte europea di elaborare fattispecie processuali coerenti con quel principio generale. Sul punto, da ultimo, con un approccio innovativo, UBERTIS, Giulio. Equità e proporzionalità versus legalità processuale: eterogenesi dei fini?, in Arch. pen., 2017, fasc. 2, p. 1 ss.; nonché, Id., Sistema di procedura penale, I, Principi generali, Giuffrè, 2017, p. 134. C. eur. dir. uomo, grande camera, 15 novembre 2016, A e B c. Norvegia, § 130, da cui è tratta anche la citazione che immediatamente segue (§ 134).

Significativamente, nella vicenda dipanatasi dinanzi alla Corte europea, sono intervenuti sei Stati terzi (Bulgaria, Repubblica ceca, Grecia, Francia, Moldavia e Svizzera), accomunati dall'intento di escludere dal divieto ex art. 4 prot. n. 7 CEDU proprio le ipotesi di duplicazioni di sanzioni nella sede penale e in quella "amministrativa": cfr. C. eur. dir. uomo, grande camera, 15 novembre 2016, A e B c. Norvegia, § 87 ss. In effetti, la Corte europea si muove, oggi, su un terreno sdrucciolevole, reso ancor più insidioso dalla crisi delle istituzioni sovranazionali, molto evidente nell'autonomo spazio giuridico dell'Unione europea: la tutela dei diritti fondamentali tende ad arretrare in rapporto alla prioritaria istanza di una "repressione efficiente" 
il fianco a severe critiche, se esaminata in rapporto al dovere degli Stati di prevenire la violazione di diritti fondamentali, ossia di dotarsi di misure strutturalmente idonee ad assicurare a priori l'osservanza dei diritti protetti ex art. 1 CEDU.

L'approccio empirico della Corte di Strasburgo fornisce un compendio (meramente esemplificativo) degli indici sintomatici della sufficiente connessione sostanziale fra procedimenti ${ }^{89}$. Che la condizione in parola possa essere soddisfatte non dipende, ovviamente, dalla discrezionalità degli organi nazionali procedenti: in un sistema a basato sulla stretta legalità formale, i rapporti fra il procedimento extrapenale e quello penale non possono essere rimessi a decisioni arbitrarie degli organi procedenti. Detto altrimenti, in difetto di disposizioni volte a introdurre meccanismi di raccordo fra il procedimento penale e quello extrapenale, le varie declinazioni del principio di legalità (sul versante penale, gli artt. 3, 25 comma 2, 101 comma 2 e 111 comma 1 Cost.; su quello amministrativo, l'art. 97 Cost.) impediscono di "forzare" la disciplina positiva, attraverso l'elaborazione ad opera della prassi di criteri extralegali ${ }^{90}$. Con riguardo, poi, alla sufficiente connessione temporale, la Corte europea non riesce, comunque, ad individuare un criterio univoco, capace di determinare quando il requisito sia integrato: la relativa individuazione è rimessa, in

propugnata da Stati nazionali sempre meno inclini a cedere porzioni di sovranità nell'ambito penale.

89 C. eur. dir. uomo, grande camera, 15 novembre 2016, A e B c. Norvegia, $\S 132-133$. La Corte europea evidenzia come essa sia assicurata, tra l'altro, quando il materiale probatorio raccolto in una sede possa essere (e sia effettivamente) impiegato nell'altra oppure quando la sanzione irrogata nel procedimento deciso successivamente a quello già concluso tenga conto delle sanzioni già irrogate all'esito di quest'ultimo.

90 Eppure, la Corte di cassazione mostra qui un atteggiamento lasco: in materia di rapporti fra procedimento disciplinare e procedimento penale, impone al giudice del merito, in sede di determinazione della pena, di «tenere conto della sanzione disciplinare già irrogata», con l'«obiettivo di completare il 'compendio sanzionatorio integrato', generato dalle due procedure [nella sede disciplinare e in quella penale], definendo una sanzione complessivamente proporzionata al disvalore del fatto plurioffensivo» (Cass., sez. II, 15 dicembre 2016, Pagano, in Dir. pen. cont., ed. on-line, 21 aprile 2017, p. 7, con nota E. Zuffada, disponibile in http://www.penalecontemporaneo.it, ultimo accesso il 24 agosto 2018). 
definitiva, all'ampia discrezionalità del giudice ${ }^{91}$. In breve, il ne bis in idem viene fatto dipendere da profili del tutto casuali (come, ad esempio, il momento del passaggio in giudicato di uno dei due provvedimenti), con irragionevoli disparità di trattamento ${ }^{92}$.

Con ciò, la Corte europea genera un autentico paradosso: l'intento di assicurare la certezza del diritto e di assegnare al ne bis in idem una sfera operativa prevedibile dal cittadino fa scaturire un esito opposto. Si spiegano, così, le durissime le parole spese dal giudice Pinto de Albuquerque, nell'articolare l'unica, robustamente argomentata, dissenting opinion alla sentenza: «il ne bis in idem ha perso la propria natura di garanzia pro persona, essendo stato trasformato dalla Corte [europea] in un rigido strumento pro auctoritate ${ }^{93}$.

Venendo all'autonomo ambito del diritto dell'Unione europea, la Corte di Giustizia aveva già assegnato alla garanzia dell'art. 50 CDFUE l'identica portata dell'art. 4 prot. n. 7 CEDU, nel significato datogli dalla Corte di Strasburgo sino alla già rammentata sentenza della grande camera nel caso A e B contro Norvegia ${ }^{94}$. Proprio l'autorevolezza ed il rilievo sul

91 Già prima della sentenza nel caso A e B c. Norvegia, la Corte europea escludeva la violazione dell'art. 4 prot. n. 7 CEDU quando - svolti parallelamente due procedimenti - il secondo si fosse concluso dopo la definizione del primo, limitando, così, l'operatività della garanzia all'ipotesi in cui il secondo procedimento fosse stato instaurato dopo che il provvedimento conclusivo del primo fosse divenuto irrevocabile: cfr., ad esempio, C. eur. dir. uomo, sez. V, 27 novembre 2014, Lucky Dev c. Svezia, § 59; sez. IV, 17 febbraio 2015, Boman c. Finlandia, § 40 ss.

92 In effetti, neppure l'argine del giudicato potrebbe offrire un sicuro riparo per scongiurare il rischio della seconda punizione, perché esplicherebbe un effetto preclusivo solo quando il secondo procedimento non fosse stato neppure instaurato al momento dell'irrevocabilità del provvedimento pronunciato nell'unico procedimento: cfr. FASOLIN, Silvia. Conflitti di giurisdizione e ne bis in idem europeo, Cedam, 2015, p. 81.

93 In breve, si sarebbero «aperte le porte a un inedito sistema punitivo, sul modello del Leviatano, basato su una pluralità di procedimenti punitivi statali, strategicamente connessi e allestiti per ottenere il più ampio effetto repressivo possibile» [Dissenting opinion del giudice Pinto de Albuquerque (§ 79)].

94 Cfr., per tutte, C. giust. UE, grande camera, 26 febbraio 2013, C-617/10, Åklagaren c. Hans Åkerberg Fransson, § 16 ss. V. anche, C. giust. UE, sez. III, 23 dicembre 2009, C-45/08, Spector Photo Group e Chris Van Raemdonck c. CBFA, $\S 30$ ss. Va precisato come, per C. giust. UE, sez. IV, 5 aprile 2017, Orsi (C217/15) e Baldetti (C-350/15), il ne bis in idem di cui all'art. 4, prot. n. 7, CEDU 
piano della politica criminale della presa di posizione della grande camera della Corte europea aveva, però, indotto a paventare che quella pronuncia avrebbe potuto generare un effetto di ritorno, influenzando un revirement della Corte di giustizia sulla portata dell'art. 50 CDFUE, «in nome di una più efficace tutela de[gli] interessi finanziari» dell'Unione europea ${ }^{95}$.

Benché vi fossero solidi argomenti capaci di scongiurare interpretazioni restrittive dell'art. $50 \mathrm{CDFUE}^{96}$, la Corte di giustizia è da ultimo pervenuta ad introdurre un'ulteriore rimodulazione, in chiave restrittiva, della portata oggettiva del ne bis in idem, benché l'Avvocato generale avesse

e 50 CDFUE non operi nel procedimento penale promosso nei confronti di una persona fisica per il reato di omesso versamento dell'IVA di cui all'art. 10-ter d.lgs. n. 74 del 2000, quando la sanzione amministrativa tributaria per lo stesso fatto fosse già stata irrogata ad una società dotata di personalità giuridica. Sulla pronuncia, SCOLETTA, Marco. Ne bis in idem e doppio binario in materia tributaria: legittimo sanzionare la società e punire il rappresentante legale per lo stesso fatto, in Dir. pen. cont., ed. on-line, 10 aprile 2017, disponibile in http://www. penalecontemporaneo.it, ultimo accesso il 24 agosto 2018.

95 Così, ancor prima della decisione della sentenza della Grande camera della Corte europea, VIGANÒ, Francesco. A never-ending story? Alla Corte di Giustizia dell'Unione Europea la questione della compatibilità tra ne bis in idem e doppio binario sanzionatorio in materia, questa volta di abusi di mercato, in Dir. pen. cont., ed. on-line, 17 ottobre 2016, disponibile in http://www.penalecontemporaneo. it, ultimo accesso il 24 agosto 2018; nonché, dopo la pronuncia in parola, SCOLETTA, Marco. Ne bis in idem e doppio binario in materia tributaria: legittimo sanzionare la società e punire il rappresentante legale per lo stesso fatto, cit.

In breve, venivano in gioco, da un lato, i principi della riserva di legge e della legalità processuale, protetti dall'art. 52 CDFUE [cfr. PANZAVOLTA, Michele. Legalità e proporzionalità nel diritto penale processuale, in MASTROIANNI, Roberto, POLLICINO, Oreste, ALLEGREZZA, Silvia, PAPPALARDO, Fabio, RAZZOLINI, Orsola (a cura di). Carta dei diritti fondamentali dell'Unione europea, cit., pp. 1005-1006]; dall'altro, il soddisfacimento del principio della "maggior tutela" ex art. 53 CDFUE che scongiura un'indebita compressione delle garanzie, poiché, se è vero che la portata dei diritti riconosciuti dalla Carta di Nizza deve corrispondere a quella assicurata dalle norme della Convenzione europea, il principio della tutela equivalente ex art. 52 comma 3 CDFUE non comporta che l'equivalenza debba essere realizzata verso il basso, interpretando in chiave antiletterale e restrittiva l'art. 50 CDFUE. Per un inquadramento generale del problema, qui appena accennato, cfr. DI PAOLA, Cristina. Carta dei diritti fondamentali dell'Unione europea, in GIARDA, Angelo, SPANGHER, Giorgio (diretto da). Codice di procedura penale commentato, tomo I, Wolters Kluwer, Milano, 2017, p. 185 ss.; nonché, volendo, anche per ulteriori riferimenti, CASSIBBA, Fabio. Disorientamenti giurisprudenziali in tema di ne bis in idem e procedimenti paralleli, cit., p. 1098 ss. 
concluso nel senso d'irrobustire la garanzia e di renderne nitidi i confini ${ }^{97}$. I giudici di Lussemburgo - se non hanno recepito l'elastico criterio della stretta connessione essenziale elaborato dalla Corte europea - hanno comunque offerto una lettura dell'art. 50 CDFUE non strettamente ancorata al dato positivo perché tutta improntata ad assicurare una tutela agli interessi finanziari dell'Unione europea. Più precisamente, dopo aver ribadito, in linea generale, che l'art. 50 CDFUE non vieta di per sé il doppio binario sanzionatorio, la grande sezione della Corte di Giustizia ha ritenuto che la condanna in sede penale divenuta irrevocabile precluda di svolgere, nei confronti della stessa persona e per il medesimo fatto, un procedimento volto alla irrogazione di una sanzione amministrativa pecuniaria avente natura sostanzialmente penale, sempre che - ecco la clausola flessibile limitativa della garanzia - la condanna penale irrevocabile, «tenuto conto del danno causato alla società dal reato commesso, sia idonea a reprimere tale reato in maniera efficace, proporzionata e dissuasiva» ${ }^{98}$. La medesima grande sezione ha, inoltre, affermato, in prospettiva speculare, che - una

97 Sull'argomento, VIGANÒ, Francesco. Le conclusioni dell'Avvocato generale nei procedimenti pendenti in materia di ne bis in idem tra sanzioni penali e amministrative in materia di illeciti tributari e abusi di mercato, in Dir. pen. cont., ed. on-line, 18 settembre 2017, disponibile in http://www.penalecontemporaneo.it, ultimo accesso il 24 agosto 2018; nonché, volendo, CASSIBBA, Fabio. Disorientamenti giurisprudenziali in tema di ne bis in idem e procedimenti paralleli, cit., p. 1098 ss.

98 Così, con riguardo al doppio binario sanzionatorio in materia di manipolazioni di mercato, CGUE, grande sezione, sent. 20 marzo 2018 nella causa C-537/16, Garlsson Real Estate SA, Stefano Ricucci, Magiste International SA c. Commissione Nazionale per la Società e la Borsa (Consob). Sulla pronuncia in parola e su quelle citate nelle note che immediatamente seguono, cfr., anche per ulteriori riferimenti, BINDI, Elena, PISANESCHI, Andrea. Corte di giustizia: il doppio binario nei reati di market abuse è illegittimo e l'art. 50 della Carta europea dei diritti fondamentali è norma direttamente applicabile, in Rivista AIC, 2012, fasc. 2, p. 1 ss.; CONSULICH, Federico; GENONI, Carolina. L'insostenibile leggerezza del ne bis in idem. Le sorti de divieto di doppio giudizio e doppia punizione, tra diritto eurounitario e diritto convenzionale, in Giur. pen. web, 2018, n. 4, p. 1; GALLUCCIO, Alessandra. La grande sezione della Corte di Giustizia si pronuncia sulle attese questioni pregiudiziali in materia di ne bis in idem, in Dir. pen. cont., ed. on-line, 21 marzo 2018, disponibile in http://www.penalecontemporaneo.it, ultimo accesso il 24 agosto 2018; NASCIMBENE, Bruno. Ne bis in idem, diritto internazionale, diritto europeo, cit.; SANTORIELLO, Ciro. Il ne bis in idem nella giurisprudenza della Corte di Giustizia UE: "così è se vi pare" (o almeno se così riterrà il giudice nazionale), in 
volta inflitta in via definitiva ad una persona una sanzione amministrativa avente natura penale - è consentito avviare nei confronti della medesima persona un procedimento penale per gli medesimi fatti, purché sussista «un obiettivo interesse generale tale da giustificare [il] cumulo di sanzioni» -, siano previste norme capaci di coordinare i due procedimenti in modo tale da limitare «a quanto strettamente necessario l'onere [per gli interessati derivante dal] cumulo di procedimenti» e di «garantire che la severità del complesso delle sanzioni imposte sia limitata a quanto ... strettamente necessario rispetto alla gravità del reato», toccando al giudice nazionale accertare la sussistenza, in concreto, di tali presupposti ${ }^{99}$. Infine, la sentenza definitiva di assoluzione, resa in ambito penale, non preclude la prosecuzione di un procedimento inteso alla irrogazione di una sanzione amministrativa pecuniaria di natura penale quando i fatti «sulla base dei quali era stato parimenti avviato tale procedimento ... non erano provati» ${ }^{100}$.

Nel complesso, prevale oggi anche del diritto dell'Unione Europea una prospettiva limitativa della portata del divieto di secondo giudizio, sbilanciata sulla sussistenza di clausole flessibili, capaci di giustificare la duplicazione di procedimenti per il medesimo fatto e tutti accomunati dall'intento di assicurar una repressione effettiva e proporzionata a quelle condotte che offendono gli interessi finanziari dell'Unione Europea. In altre parole, anche alla luce della giurisprudenza della Corte di giustizia, la considerazione del ne bis in idem come diritto fondamentale è destinata a cedere il passo a finalità di politica criminale e di armonizzazione dei sistemi penali ${ }^{101}$.

Il Penalista, ed. on-line, 23 aprile 2018, disponibile in http://www.ilpenalista. it, ultimo accesso il 24 agosto 2018.

99 Così, con riguardo al doppio binario sanzionatorio in materia di omesso versamento dell'imposta sul valore aggiunto, CGUE, grande sezione, sent. 20 marzo 2018, nella causa C-524/15, Luca Menci.

${ }^{100}$ Così, con riguardo al doppio binario sanzionatorio in materia di abuso di informazioni privilegiate e di manipolazioni di mercato, CGUE, grande sezione, sent. 20 marzo 2018, nelle cause riunite C-596/16 e C-597/16, Enzo Di Puma c. Commissione Nazionale per la Società e la Borsa (Consob).

101 In effetti, se persino nell'ambito convenzionale stenta ad affermarsi - senza pericolose regressioni - l'assunto secondo cui «una Corte investita della tutela dei diritti dell'uomo non deve scendere sotto il livello di protezione dalla stessa già garantito» (Dissenting opinion dei giudici Sajó e Laffranque, nel caso deciso da Corte e.d.u., grande camera, 13 settembre 2016, Ibrahim e altri c. Regno unito, § 2), a fortiori, è elevato il rischio che analoghe restrizioni 
In conclusione, dal punto di vista degli ordinamenti sovranazionali, la portata oggettiva del ne bis in idem in rapporto al doppio binario sanzionatorio resta tutt'altro che prevedibile. L'operatività della garanzia resta esposta ai criteri di pura creazione giurisprudenziale, ispirati al massimo pragmatismo, privi della necessaria determinatezza e, dunque, di generare irragionevoli disparità di trattamento. D'altro canto, è decisivo notare che i criteri in parola non sono neppure davvero idonei a regolare la portata del ne bis in idem, perché per definizione si basano su valutazioni che possono essere utilmente compiute solo ex post, ossia dopo la definizione dei diversi procedimenti, quando ormai la violazione del divieto potrebbe essersi già consumata.

\subsection{NE BIS IN IDEM E DOPPIO BINARIO SANZIONATORIO NELL'ORDINAMENTO NAZIONALE}

A fronte delle decisioni prese dalle Corti sovranazionali appena rammentate, v’è da chiedersi quali siano le vie percorribili per assicurare una sfera operativa del ne bis in idem nazionale ex art. 649 c.p.p. che risulti coerente con l'art. 50 CDFUE, da un lato, e con l'art. 4 prot. 7 CEDU, dall'altro. Il quesito è ineludibile, perché «[s]petta ... al giudice nazionale, che deve confrontarsi con un sistema integrato delle fonti costituzionali, comunitarie e internazionali ..., assicurare la costante tutela dei diritti fondamentali», fra i quali rientra, appunto, quello del ne bis in idem ${ }^{102}$. Più precisamente, occorre adeguare la sfera operativa del divieto di bis in idem alle garanzie europee in materia quando - divenuto definitivo il provvedimento amministrativo (ma sostanzialmente penale) - sia ancora

vengano attuate dalla Corte di Giustizia: qui, la tutela dei diritti fondamentali è destinata a rilevare nella misura in cui possa risultare funzionale al - o non contrastante col - perseguimento delle finalità d'integrazione europea (così, KOSTORIS, Roberto Ettore. La tutela dei diritti fondamentali, cit., p. 86).

102 Così, Cass., sez. VI, 15 novembre 2016, Resnelli, cit., p. 10. Sulla portata della pronuncia, con particolare riguardo alla diretta applicabilità dell'art. 50 CDFUE da parte del giudice nazionale, GITTARDI, Irene. La miccia è accesa: la Corte di cassazione fa diretta applicazione dei principi della Carta di Nizza in materia di ne bis in idem, in Dir. pen. cont., ed. on-line,7 aprile 2017, p. 1 ss., disponibile in http://www.penalecontemporaneo.it, ultimo accesso il 24 agosto 2018. 
pendente, per il medesimo fatto e nei confronti della medesima persona, il procedimento formalmente penale. Non va, però, sottaciuto il rischio che i giudici nazionali - proprio sulla scorta delle suggestioni delle decisioni prese in sede sovranazionale, prima ancora che di una loro autentica efficacia vincolante ${ }^{103}$ - recepiscano, acriticamente, i criteri dei giudici europei, aggirando il divieto di secondo giudizio ${ }^{104}$.

${ }^{103}$ Quanto alle sentenze della Corte europea dei diritti dell'uomo, a seguito delle sent. cost. n. 348 e 349 del 2007, è sorto, nella dottrina processualpenalistica, un acceso dibattito fra chi riconosce la fondatezza della posizione della Corte costituzionale, reputando vincolanti le interpretazioni fornite dalla Corte europea a proposito dei requisiti dell'equità processuale di cui all'art. 6 Conv. eur. dir. uomo, che divengono così pure esse parametro interposto nello scrutinio di costituzionalità alla luce dell'art. 117 comma 1 Cost. (cfr. UBERTIS, Giulio. La "Rivoluzione d'ottobre" della Corte costituzionale e alcune discutibili reazioni, in Cass. pen., 2012, p. 19 ss.), e chi, invece, la nega (cfr. FERRUA, Paolo. L'interpretazione della Convenzione europea dei diritti dell'uomo e il preteso monopolio della Corte di Strasburgo, in Proc. pen. giust., 2011, p. 121). Da ultimo, per C. cost., sent. 26 marzo 2015 n. 49, in Giur. cost., 2015, p. 408, il vincolo interpretativo per il giudice nazionale scatta solo in rapporto al «'diritto consolidato', generato dalla giurisprudenza europea», non sussistendo, invece, alcun dovere di interpretazione conforme a fronte di pronunce europee che non siano espressive di un orientamento che oramai è divenuto definitivo». Sull'ampio dibattito generato da tale ultima pronuncia v., fra i molti e da angoli visuali diversi, MANES, Vittorio. La "confisca senza condanna" al crocevia tra Roma e Strasburgo: il nodo della presunzione di innocenza; PULITANÒ, Domenico. Due approcci opposti sui rapporti fra Costituzione e CEDU in materia penale. Questioni lasciate aperte da Corte cost. n. 49/2015; VIGANÒ, Francesco. La Consulta e la tela di Penelope, tutti in Dir. pen. cont., ed. on-line, 30 marzo 2015, disponibile in http://www.penalecontemporaneo.it, ultimo accesso il 24 agosto 2018. Più in generale, sull'evoluzione della giurisprudenza costituzionale in materia, cfr., per tutti, FERRUA, Paolo. La prova nel processo penale, I, Struttura e procedimento, Giappichelli, 2017, p. 297 ss. Quanto alle sentenze della Corte di Giustizia, a fronte di chi sostiene la portata erga omnes della pronuncia sulla questione pregiudiziale (così: ADAM, Roberto; TIZZANO, Antonio. Lineamenti dell'Unione europea, Giappichelli, 2016, p. 262; TESAURO, Giuseppe. Manuale di diritto dell'Unione europea, Cedam, 2012, p. 324) altri ritengono che non sorga nei giudizi nazionali diversi da quello in cui sono state sollevate le questioni un vincolo del giudice ad attenersi alla soluzione offerta dalla Corte di Giustizia (così, GAJA, Giorgio; ADINOLFI, Adelina. Introduzione al diritto dell'Unione europea, Laterza, 2010, p. 116).

${ }^{104}$ V., ad esempio, nella giurisprudenza di legittimità, Cass., sez. III, 22 settembre 2017, n. 6993, in Dir. pen. cont. ed. on-line del 16 marzo 2018, con nota di TRIPODI, Andrea Francesco; nella giurisprudenza di merito, Trib. Milano, sez. I, 6 dicembre 2016, D.F. e altri, in Dir. pen. proc., 2017, p. 514, con nota di 
Dal punto di vista metodologico, a legislazione invariata, l'adeguamento ai canoni europei potrebbe, in linea d'ipotesi, attuarsi (e già si è tentato di attuarlo da parte della giurisprudenza) attraverso varie soluzioni. Più analiticamente, s'è proposto: $a$ ) di ricorrere ad un'interpretazione conforme e convenzionalmente orientata dell'art. 649 c.p.p.; $b$ ) di sollevare una questione di legittimità dell'art. 649 c.p.p., per contrasto con l'art. 117, comma 1, Cost., invocando come norma interposta l'art. 4 prot. n. 7 CEDU; $c$ ) di fare diretta applicazione nell'ordinamento interno dell'art. 50 CDFUE oppure dell'art. 4 prot. n. 7 CEDU $^{105}$. Nessuna delle soluzioni prospettate risulta, peraltro, soddisfacente.

a) Va, anzitutto, escluso che la soluzione possa consistere in un'interpretazione conforme e convenzionalmente orientata dell'art. 649 c.p.p. sulla premessa che il riferimento alle decisioni penali irrevocabili ivi considerate includa anche quelle emesse da un'autorità extrapenale, purché operino in materie sostanzialmente penali, secondo i criteri Engel $^{106}$. L'operazione - avversata sul piano metodologico anche dalla

MANACORDA, Stefano, ambedue disponibili in http://www.penalecontemporaneo.it, ultimo accesso il 24 agosto 2018.

${ }^{105}$ Sul tema v., fra gli altri, BONTEMPELLI, Manfredi. Il doppio binario sanzionatorio in materia tributaria e le garanzie europee ( $f r a$ ne bis in idem processuale e ne bis in idem sostanziale), in Arch. pen., 2015, fasc. 2, p. 126 ss.; CAIANIELLO, Michele. Ne bis in idem e illeciti tributari per omesso versamento dell'Iva: il rinvio della questione alla Corte costituzionale, cit., p. 6 ss.; LAVARINI, Barbara. Il ne bis in idem convenzionale e "doppio binario" sanzionatorio: il problema del "doppio giudicato", in Leg. pen., ed. on-line, 14 marzo 2016, p. 2 ss., disponibile in http://www.legisazionepenale.it, ultimo accesso il 24 agosto 2018; Ead., Corte europea dei diritti umani e ne bis in idem: la crisi del "doppio binario" sanzionatorio, cit., p. 87-88; MAZZA, Oliviero. L'insoste $\neg$ nibile convivenza fra ne bis in idem europeo e doppio binario sanzionatorio per i reati tributari, cit., p. 1040 ss.; VIGANÒ, Francesco. Doppio binario sanzionatorio e ne bis in idem: verso una diretta applicazione dell'art. 50 della Carta?, cit., p. 234 ss.

${ }^{106}$ In questo senso v., invece, pur con diverse sfumature, CAIANIELLO, Michele. Ne bis in idem e illeciti tributari per omesso versamento dell'Iva: il rinvio della questione alla Corte costituzionale, cit., p. 6 ss.; FLICK, Giovanni Maria, NAPOLEONI, Valerio. Cumulo tra sanzioni penali e amministrative: doppio binario o binario morto? "Materia penale", giusto processo e ne bis in idem nella sentenza della Corte EDU, 4 marzo 2014, sul market abuse, in Rivista AIC, 2014, fasc. 3, p. 10; VIGANÒ, Francesco. Doppio binario sanzionatorio e ne bis in idem: verso una diretta applicazione dell'art. 50 della Carta?, cit., p. 234 ss. In giurisprudenza, Cass., sez. III, 21 aprile 2016, Di Stasi, in Dir. pen. 
Corte costituzionale ${ }^{107}$ - è «apertamente contraria alle regole dell'ermeneutica» ${ }^{108}$ : forza il dato letterale della previsione per assegnarle un significato del tutto estraneo. D'altro canto, neppure l'elevato prezzo di legittimare un diritto giurisprudenziale ampiamente creativo assicurerebbe una soluzione alle ipotesi in cui a passare in giudicato per primo fosse il provvedimento formalmente penale: l'autorità amministrativa, fuori dai casi in cui il codice di procedura penale o leggi speciali regolano l'efficacia extrapenale del giudicato, non potrebbe fare applicazione dell'art. 649 c.p.p., nel suo significato conforme o convenzionalmente orientato.

b) Miglior sorte non avrebbe la soluzione della questione di legittimità costituzionale dell'art. 649 c.p.p., sollevata per un contrasto con l'art. 4 prot. n. 7 CEDU (così come interpretato dalla Corte europea), in rapporto all'art. 117 comma 1 Cost., allo scopo di renderlo operante anche quando uno dei due procedimenti abbia natura sostanzialmente penale ${ }^{109}$. Una declaratoria d'illegittimità si scontrerebbe con diversi controlimiti costituzionali, destinati a valere almeno nell'ipotesi in cui il provvedimento amministrativo fosse diventato irrevocabile per primo e dispiegasse un effetto preclusivo sulla vicenda penale, ancora pendente ${ }^{110}$. Verrebbero in gioco le garanzie dell'equo processo penale ex art. 111

cont., ed. on-line, 11 luglio 2016, con nota di VIGANÒ, Francesco. disponibile in http://www.penalecontemporaneo.it, ultimo accesso il 24 agosto 2018; Trib. Brindisi, 17 ottobre 2014, in Dir. pen. proc., 2015, p. 438 ss., con nota di DI BITONTO, Maria Lucia.

${ }^{107}$ Cfr. C. cost., sent. 11 marzo 2011 n. 80, in Giur. cost., 2011, 1224, sia pur resa in un ambito diverso da quello qui considerato.

108 MAZZA, Oliviero. L'insostenibile convivenza fra ne bis in idem europeo e doppio binario sanzionatorio per i reati tributari, cit., p. 1040. V. anche, LAVARINI, Barbara. Corte europea dei diritti umani e ne bis in idem: la crisi del "doppio binario" sanzionatorio, cit., p. 87; DE AMICIS, Gaetano. Ne bis in idem e "doppio binario" sanzionatorio: prime riflessioni sugli effetti della sentenza "Grande Stevens" nell'ordinamento italiano, cit., p. 201 ss.

109 In senso favorevole, v., invece, LAVARINI, Barbara. Corte europea dei diritti umani e ne bis in idem: la crisi del "doppio binario" sanzionatorio, cit., p. 87; contra, DI BITONTO, Maria Lucia. Una singolare applicazione dell'art. 649 c.p.p., cit., p. 447.

${ }^{110}$ Così, MAZZA, Oliviero. L'insostenibile convivenza fra ne bis in idem europeo e doppio binario sanzionatorio per i reati tributari, cit., p. 1040-1041. 
Cost. ${ }^{111}$, l'obbligatorietà dell'azione penale ex art. 112 Cost. ${ }^{112}$, nonché la presunzione di innocenza ex art. 27 comma 2 Cost.

Non si dimentichi, poi, che il tenore dell'art. 4 prot. n. 7 CEDU non implica indefettibilmente il divieto di un secondo giudizio: s'è detto ${ }^{113}$ che la norma convenzionale non è violata nel caso in cui il soggetto - già irrevocabilmente sanzionato nella sede amministrativa - sia poi prosciolto in quella formalmente penale ${ }^{114}$.

c) Non sarebbe, infine, risolutivo individuare la soluzione nella diretta applicazione dell'art. 4 prot. n. 7 CEDU in luogo dell'art. 649 c.p.p.,

${ }^{111} \mathrm{Si}$ assegnerebbe efficacia preclusiva nella sede penale ad un provvedimento emesso da un'autorità formalmente extrapenale in assenza di tali garanzie. Nel senso che l'«ordinamento nazionale può apprestare garanzie ulteriori rispetto a quelle convenzionali, riservandole alle sole sanzioni penali, così come qualificate dall'ordinamento interno», cfr., da ultimo, sulla scia di un indirizzo consolidato, C. cost., 24 febbraio 2017, n. 43 (§ 4.1 del Considerato in diritto), poiché «ciò che per la giurisprudenza europea ha natura "penale" deve essere assistito dalle garanzie che la stessa ha elaborato per la "materia penale"; mentre solo ciò che è penale per l'ordinamento nazionale beneficia degli ulteriori presìdi rinvenibili nella legislazione interna» ( $\$ 3.4$ del Considerato in diritto).

112 S'imporrebbe una declaratoria d'improcedibilità dell'azione penale sul presupposto che il procedimento amministrativo si sia concluso per primo, con conseguente violazione dell'art. 3 Cost.

113 V. supra, § 1.

${ }^{114}$ In ogni caso, resterebbe aperta la questione dell'attribuzione della natura sostanzialmente penale del procedimento. Certo, varrebbero i criteri Engel, che, tuttavia, per quanto consolidati nella giurisprudenza di Strasburgo, non sono idonei a circoscrivere la portata del divieto di secondo giudizio ex art. 649 c.p.p. entro un numero chiuso di procedimenti. Anzi, la portata evolutiva delle fattispecie convenzionali non esclude che, in futuro, la Corte europea possa riconoscere la natura sostanzialmente penale anche a procedimenti che oggi restano fuori dall'ambito dell'art. 4 prot. n. 7 CEDU. Sotto questo profilo, l'abbandono, da parte dell'ordinamento interno, di una concezione della garanzia del ne bis in idem ancorata ad un giudicato formalmente penale svela scenari inediti e conduce ad esiti tutt'altro che prevedibili. Si pensi, ad esempio, al rapporto fra il procedimento penale e quello disciplinare (sul tema, di recente, CORSO, Piermaria. Prospettive evolutive del ne bis in idem, cit., p. 20; DI BITONTO, Maria Lucia. Una singolare applicazione dell'art. 649 c.p.p., cit., p. 441 ss.; nella giurisprudenza di Strasburgo, da ultimo, C. eur. dir. uomo, sez. III, dec. 3 luglio 2018, Jorge Manuel Làzaro Laporta c. Spagna), prima assoggettato all'art. 649 c.p.p. dalla giurisprudenza di merito (Trib. Brindisi, sent. 17 ottobre 2014, cit., p. 438 ss.), poi escluso dalla sua portata da parte della Corte di cassazione (Cass., sez. II, 15 dicembre 2016, Pagano, cit.). 
secondo un innovativo percorso proposto dalla dottrina ${ }^{115}$. La portata normativa della previsione in parola non individua, infatti, un ambito univoco di applicabilità della garanzia del divieto di secondo giudizio. Dal punto di vista del diritto dell'Unione europea, poi, «congenitamente insufficiente» ${ }^{116}$ sul piano sistematico sarebbe la soluzione basata sulla diretta applicazione dell'art. 50 CDFUE $^{117}$ : la previsione opera solo all'interno della sfera del diritto dell'Unione europea, come pure messo in luce della stessa Corte di Giustizia ${ }^{118}$

A tal punto, non resta che riedificare il sistema attorno a due linee ricostruttive.

Da un lato, la giurisprudenza interna è chiamata a valorizzare con rigore le clausole normative di specialità: va evitata la violazione del ne bis in idem negli ambiti in cui la doppia sanzione non è legislativamente consentita $^{119}$. La soluzione implica di ritenere assorbito l'intero disvalore del

115 Cfr. VIGANÒ, Francesco. Art. 4 Prot. $n .7$ - Divieto di secondo giudizio, cit., p. 389 , sul presupposto che la previsione in parola introduca una «norma incorporata nell'ordinamento italiano in forza della legge 9 aprile $1990 \mathrm{n}$. 98 di esecuzione e autorizzazione alla ratifica [del Protocollo n. 7 CEDU, e dalla quale dovrebbe discendere l'obbligo per il giudice penale italiano di pronunciare sentenza di proscioglimento o di non luogo a procedere in presenza delle condizioni stabilite dalla giurisprudenza pertinente di Strasburgo, e dunque anche oltre le ipotesi contemplate dalla disposizione di diritto interno - l'art. 649 c.p.p. - in materia di ne bis in idem».

116 MAZZA, Oliviero. L'insostenibile convivenza fra ne bis in idem europeo e doppio binario sanzionatorio per i reati tributari, cit., p. 1041.

117 Cfr., da ultimo, VIGANÒ, Francesco. Art. 4 Prot. $n$. 7 - Divieto di secondo giudizio, cit., p. 387-388.

118 Cfr. C. giust. UE, sez. IX, 15 aprile 2015, causa C-497/14, Burzio, spec. $\S 31$, in Dir. pen. cont., ed. on-line, 8 maggio 2015, con nota di SCOLETTA, Marco. Disponibile in <http://www.penalecontemporaneo.it>, ultimo accesso: 24 agosto 2018.

119 Ad esempio, va superata l'opzione interpretativa adottata dalle Sezioni unite, a proposito dell'art. 10-bis d.lgs. n. 74 del 2000, secondo cui l'illecito amministrativo e l'illecito penale si collocano in un rapporto di progressione, che sarebbe idoneo ad escludere l'operatività del principio di specialità: $\mathrm{cfr}$. Cass., sez. un., 28 marzo 2013, Favellato, in Cass. pen., 2014, p. 54 ss., con nota di Ciraulo; Cass., sez. un., 28 marzo 2013, Romano, in Dir. pen. cont., ed. on-line, 18 settembre 2013, con nota di A. Valsecchi, disponibile in http:// www.penalecontemporaneo.it, ultimo accesso il 24 agosto 2018. Sulla crisi del principio di specialità e sul conseguente aggiramento della garanzia del ne bis in idem v., anche per ulteriori riferimenti, CARINCI, Andrea. Il principio 
fatto dalla sanzione formalmente penale, con conseguente improcedibilità dell'azione amministrativa o sua rinuncia in favore di quella penale ${ }^{120}$. Dall'altro, l'adeguamento al canone europeo del ne bis in idem rappresenta un «giardino proibito» per la giurisprudenza perché riservato al legislatore ${ }^{121}$, come pure evidenziato dalla sent. cost. n. 102 del $2016^{122}$ : occorre un complessivo intervento riformatore volto a introdurre misure strutturalmente idonee a prevenire la violazione di diritti fondamentali (artt. 1 e 46 CEDU).

Prima ancora di coinvolgere la disciplina processuale, delineando un procedimento unitario eventualmente volto all'irrogazione di entrambe le sanzioni ${ }^{123}$, è ineludibile riordinare i meccanismi sanzionatori, improntandone i rapporti al principio di specialità e irrobustendo la natura sussidiaria dell'intervento penale in senso stretto ${ }^{124}$. Solo su tale

di ne bis in idem, tra opportunità e crisi del sistema sanzionatorio tributario, in Arch. pen., 2017, fasc. 1, p. 28 ss.

120 Così, MAZZA, Oliviero. L'insostenibile convivenza fra ne bis in idem europeo e doppio binario sanzionatorio per i reati tributari, cit., p. 1042. Non si dimentichi che, dal punto di vista sistematico, il ne bis in idem sostanziale costituisce una condizione di operatività del ne bis in idem processuale, benché non sia vero il contrario, giacché la sfera dell'art. 649 c.p.p. trascende le ipotesi di concorso apparente di norme: sull'impossibilità di sovrapporre la portata del ne bis in idem sostanziale e di quello processuale, per tutti, CAPRIOLI, Francesco. Il principio del ne bis in idem, cit., p. 87 ss.; nonché, già CONSO, Giovanni. I fatti giuridici processuali penali. Perfezione ed efficacia, Giuffrè, 1955, p. 103-104. Con più diretto riferimento ai temi qui affrontati v., da ultimo, con varietà di approcci, BONTEMPELLI, Manfredi. Ne bis in idem e legalità penale nel processo per gli abusi di mercato, in Arch. pen., 2016, fasc. 2, p. 395; RANALDI, Gaetano; GAITO, Federico. Introduzione allo studio dei rapporti tra ne bis in idem sostanziale e processuale, in Arch. pen., 2017, fasc. 1, p. 103 ss.

121 Per impiegare qui - a contrario - un'espressione di FERRUA, Paolo. Un giardino proibito per il legislatore: la valutazione della prova, in Quest. giust., 1998, fasc. 3, p. 83 ss.

122 Cfr. C. cost., sent. 12 maggio 2016 n. 102, in Giur cost., 2016, p. 896 ss.

${ }^{123}$ Come messo in luce anche da C. eur dir. uomo, grande camera, sent. 15 novembre 2016, A e B c. Norvegia, § 130.

124 Cfr., con vari accenti e da vari angoli visuali, CORSO, Piermaria. Prospettive evolutive del ne bis in idem, cit., p. 25 ss.; DI BITONTO, Maria Lucia. Una singolare applicazione dell'art. 649 c.p.p., cit., p. 448; DE AMICIS, Gaetano; GAETA, Piero. Il confine di sabbia: la Corte Edu ancora di fronte al divieto del ne bis in idem, cit., p. 469 ss.; GALANTINI, Novella. Il principio del ne bis in idem tra doppio processo e doppia sanzione, in Giur. it., 2015, p. 222; LAVARINI, Barbara. Corte europea dei diritti umani e ne bis in idem: la crisi del "doppio binario" 
premessa appare ragionevole estendere la portata del ne bis in idem ex art. 649 c.p.p. a quegli ambiti in cui il legislatore ha espressamente considerato indispensabile mantenere in vita il doppio binario sanzionatorio.

Va ribadito che, in un sistema connotato dalla legalità formale, la sfera operativa del ne bis in idem processuale (a tutto tondo, "convenzionalmente" ed "eurounitariamente" plasmata) può essere costruita dal solo legislatore: in assenza di un suo intervento chiarificatore, «[n] essuno ... oggi è in grado di dire, in base agli insegnamenti europei, quale sia la corretta interpretazione dell'art. 649 c.p.p.» ${ }^{125}$. Rilievo paradossale, se si pone mente che la legalità processuale non solo è protetta dall'art. 111 comma 1 Cost., ma è - da tempo - reputata un principio generale dell'ordinamento dagli stessi giudici di Strasburgo ${ }^{126}$.

\section{Bibliografia}

AMALFITANO, Chiara, D'AMBROSIO, Raffaele. Art. 50 - Diritto a non essere giudicato o punito due volte per lo stesso reato, in MASTROIANNI, Roberto, POLLICINO, Oreste, ALLEGREZZA, SILVIA, PAPPALARDO, Fabio, RAZZOLINI, Orsola (a cura di). Carta dei diritti fondamentali dell'Unione europea, Milano: Giuffrè, 2017, p. 1031 s.

ANTOLISEI, Francesco. Manuale di diritto penale. Parte generale, XIV ed. aggiornata e integrata da CONTI, Luigi, Milano: Giuffrè, 1997, p. 215 s.

BERNARDI, Alessandro (a cura di). I controlimiti. Primato delle norme europee e difesa dei principi costituzionali, Napoli: Jovene, 2017.

BERNARDI, Alessandro, CUPELLI, Cristiano (a cura di). Il caso Taricco e il dialogo tra le Corti. L'ordinanza 24/2017 della Corte costituzionale, Napoli: Jovene, 2017.

sanzionatorio, cit., p. 86; MAZZA, Oliviero. L'insostenibile convivenza fra ne bis in idem europeo e doppio binario sanzionatorio per i reati tributari, cit., p. 1042; PROCACCINO, Angela. Il ne bis in idem dalla "certezza del diritto" alla certezza del "diritto soggettivo", in GAITO, Alfredo; CHINNICI, Daniela (a cura di). Regole europee e processo penale, Padova, 2016, p. 269; VIGANÒ, Francesco. Art. 4 Prot. $n .7$ - Divieto di secondo giudizio, cit., p. 390.

125 FERRUA, Paolo. La prova nel processo penale, I, Struttura e procedimento, cit., p. 291, nota 48 .

${ }^{126}$ Cfr. Corte e.d.u., sez. II, 22 giugno 2000, Coëme c. Belgio. 
BINDI, Elena, PISANESCHI, Andrea. Corte di giustizia: il doppio binario nei reati di market abuse è illegittimo e l'art. 50 della Carta europea dei diritti fondamentali è norma direttamente applicabile, in Rivista AIC, 2012, fasc. 2, p. $1 \mathrm{~s}$.

BONTEMPELLI, Manfredi. Il doppio binario sanzionatorio in materia tributaria e le garanzie europee ( fra ne bis in idem processuale e ne bis in idem sostanziale), em Arch. pen, 2015, fasc. 2, p. 126 s.

BONTEMPELLI, Manfredi. Ne bis in idem e legalità penale nel processo per gli abusi di mercato, em. Arch. pen., 2016, n. 2, p. 395.

BONTEMPELLI, Manfredi. La litispendenza penale, Milano: Giiuffrè, 2017.

BRANCACCIO, Matilde, FIDELBO, Giorgio. Ne bis in idem: percorsi giurisprudenziali e recenti approdi della giurisprudenza nazionale ed europea, Relazione di orientamento dell'Ufficio del Massimario penale, 21 marzo 2017, disponibile in http://www.cortedicassazione.it, ultimo accesso il 24 agosto 2018, p. 1 s.

BUZZELLI, Silvia. Processo penale europeo, in Enc. dir., Annali, II, tomo I, Milano: Giuffrè, 2008, p. 701 s.

BUZZELLI, Silvia. Procedimenti paralleli, spazio di giustizia, Unione europea: il contesto normativo e gli aspetti problematici, em Archivio penale, 2012, fasc. 1, p. $1 \mathrm{~s}$.

CAIANIELLO, Michele. Ne bis in idem e illeciti tributari per omesso versamento dell'Iva: il rinvio della questione alla Corte costituzionale, em Dir. pen. cont., ed. on-line del 15 maggio 2015, p. 1 s., consultabile all'indirizzo http://penalecontemporaneo. it, ultimo accesso il 24 agosto 2018.

CALLARI, Francesco. La firmitas del giudicato penale: essenza e limiti, Milano: Giuffrè, 2009.

CAPRIOLI, Francesco. Il principio del ne bis in idem, in CAPRIOLI, Francesco, VICOLI, Daniele, Procedura penale dell'esecuzione, Torino: Giappichelli, 2011, p. 67 s.

CAPRIOLI, Francesco. Giudicato e illegalità della pena: riflessioni a margine di una recente sentenza della Corte costituzionale, in BARGIS, Marta (a cura di), Studi in ricordo di Maria Gabriella Aimonetto, Milano: Giuffré, 2013, p. 263 s.

CAPRIOLI, Francesco. Il giudice e la legge processuale: il paradigma rovesciato, em Ind. pen., 2017, p. 967 s.

CARINCI, Andrea. Il principio di ne bis in idem, tra opportunità e crisi del sistema sanzionatorio tributario, em Arch. pen., 2017, fasc. 1, p. 28 s.

CASSESE, Sabino. I tribunali di Babele. I giudici alla ricerca di un nuovo ordine globale, Roma: Donzelli, 2009. 
CASSIBBA, Fabio; COLELLA, Angela. Art. 3 - Proibizione della tortura, in UBERTIS, Giulio; VIGANÒ, Francesco (a cura di). Corte di Strasburgo e giustizia penale, Torino: Giappichelli, 2016, p. 64 s.

CASSIBBA, Fabio. L'imputazione e le sue vicende, in UBERTIS, Giulio; VOENA, Giovanni Paolo (diretto da). Trattato di procedura penale, vol. XXXI, Milano: Giuffrè, 2016.

CASSIBBA, Fabio. Disorientamenti giurisprudenziali in tema di ne bis in idem $e$ procedimenti paralleli, em Proc. pen. giust., 2017, fasc. 6, p. 1098 s.

CONSO, Giovanni. I fatti giuridici processuali penali. Perfezione ed efficacia, Milano: Giuffrè, 1955.

CONTI, Carlotta. La preclusione nel processo penale, Milano: Giuffrè, 2014.

CONSULICH, Federico; GENONI, Carolina. L'insostenibile leggerezza del ne bis in idem. Le sorti de divieto di doppio giudizio e doppia punizione, tra diritto eurounitario e diritto convenzionale, em Giur. pen. web, 2018, fasc. 4, p. 1 ss., disponibile in http:// www.giurisprudenzapenale.it, ultimo accesso il 24 agosto 2018.

CORDERO, Franco. Considerazioni sul principio di identità del fatto, em Riv. it. dir. proc. pen., 1958, p. $936 \mathrm{~s}$.

CORDERO, Franco. Procedura penale, Milano: Giuffrè, 2012.

CORSO, Piermaria. Prospettive evolutive del ne bis in idem, em Arch. pen., 2017, fasc. 1 , p. $13 \mathrm{~s}$.

DANIELE, Marcello. Profili sistematici della sentenza di non luogo a procedere, Torino: Giappichelli, 2005.

DANIELE, Marcello. La triangolazione delle garanzie processuali tra Diritto dell'Unione europea, Cedu e sistemi nazionali, em Dir. pen. cont., ed. on-line del 6 aprile 2016, p. 1 ss., in htpp://www.penalecontemporaneo.it, ultimo accesso il 24 agosto 2018.

DE AMICIS, Gaetano. Ne bis in idem e "doppio binario" sanzionatorio: prime riflessioni sugli effetti della sentenza "Grande Stevens" nell'ordinamento italiano, em Dir. pen. cont. - Riv. trim., 2014, fasc. 3-4, p. 203 ss., disponibile in https://www. penalecontemporaneo.it, ultimo accesso il 24 agosto 2018.

DE AMICIS, Gaetano, GAETA, Piero. Il confine di sabbia: la Corte Edu ancora di fronte al divieto del ne bis in idem, em Cass. pen., 2017, p. 469 s.

DELLA MONICA, Giuseppe. Ne bis in idem, em Gaito, Alfredo (a cura di). I principi europei del processo penale, Roma: Dike, 2016, p. $331 \mathrm{~s}$.

DE LUCA, Giuseppe. Giudicato, Diritto processuale penale, em Enc. giur. Treccani, XV, 1989, p. 1 s. 
DI BITONTO, Maria Lucia. Una singolare applicazione dell'art. 649 c.p.p., em Dir. pen. proc., 2015, p. $441 \mathrm{~s}$.

DI GIOVINE, Ombretta. Come la legalità europea sta riscrivendo quella nazionale. Dal primato della legge a quello dell'interpretazione, em Dir. pen. cont. - Riv. trim., 2013, fasc. 1, p. 159 ss., consultabile all'indirizzo https://www.penalecontemporaneo. it, ultimo accesso il 24 agosto 2018;

DINACCI, Filippo Raffale. Interpretazione "europeisticamente" orientata tra fonti normative e resistenze giurisprudenziali, in GAITO, Alfredo (a cura di). I princìi europei del processo penale, Roma: Dike, 2016, p. 58 s.

DI PAOLA, Cristina. Carta dei diritti fondamentali dell'Unione europea, in GIARDA, Angelo, SPANGHER, Giorgio (diretto da). Codice di procedura penale commentato, tomo I, Milano: Wolters Kluwer, 2017, p. 185 s.

DOMINIONI, Oreste. L'esercizio dell'azione penale (l'imputazione) (1970), in Le parti nel processo penale. Profili sistematici e problemi, Milano: Giuffrè, 1985.

DOSI, Ettore. La sentenza penale di proscioglimento, Milano: Giuffrè, 1955.

DOVA, Massimiliano. Ne bis in idem e reati tributari: una questione ormai ineludibile, em Dir. pen. cont., ed on-line, 11 dicembre 2014, disponibile in https://www. penalecontemporaneo.it, ultimo accesso il 24 agosto 2018.

FALCINELLI, Daniela. Il fatto di reato sullo sfondo del ne bis in idem nazional-europeo, em Arch. pen., 2017, fasc. 1, p. 69 s.

FASOLIN, Silvia. Conflitti di giurisdizione e ne bis in idem europeo, Padova: Cedam, 2015.

FERRAJOLI, Luigi. Diritti fondamentali. Un dibattito teorico, Roma-Bari: Laterza, 2008.

FERRUA, Paolo. Un giardino proibito per il legislatore: la valutazione della prova, em Quest. giust., 1998, fasc. 3, p. 83 s.

FERRUA, Paolo. La sentenza costituzionale sul caso Eternit: il ne bis in idem tra diritto vigente e diritto vivente, em Cass. pen., 2017, p. 78 s.

FIANDACA, Giovanni. Fatto nel diritto penale, em D. disc. pen., vol. V, Torino: Utet, 1991, p. $153 \mathrm{~s}$.

FIMIANI, Paolo. Market abuse e doppio binario sanzionatorio dopo la sentenza della Corte E.D.U., Grande camera, 15 novembre 2016, A e B c. Norvegia, em Dir. pen. cont., ed. on-line, 8 febbraio 2017, p. 1 ss., disponibile in https://www.penalecontemporaneo.it, ultimo accesso il 24 agosto 2018.

FLICK, Giovanni Maria, NAPOLEONI, Valerio. Cumulo tra sanzioni penali e amministrative: doppio binario o binario morto? "Materia penale", giusto processo e ne 
bis in idem nella sentenza della Corte EDU, 4 marzo 2014, sul market abuse, em Rivista AIC, 2014, fasc. 3, p. 10 s.

GALATINI, Novella. Il divieto del doppio processo per lo stesso fatto come diritto della persona, em Riv. it. dir. proc. pen., 1981, p. 101 s.

GALATINI, Novella. Il principio del ne bis in idem internazionale nel processo penale, Milano: Giuffrè, 1984.

GALATINI, Novella. Il principio del ne bis in idem tra doppio processo e doppia sanzione, em Giur. it., 2015, p. 217 s.

GALATINI, Novella. Postilla ad uno scritto in tema di ne bis in idem, em Dir. pen. cont., ed. on-line del 30 gennaio 2017, p. 1 ss., consultabile all'indirizzo http:// www.penalecontemporaneo.it, ultimo accesso il 24 agosto 2018

GALANTINI, Novella. Il 'fatto' nella prospettiva del divieto di secondo giudizio, em Riv. it. dir. proc. pen., 2017, p. 1205 s.

GALLIANI, Davide. Su mestiere del giudice, tra Costituzione e Convenzione, em http://www.giurcost.org, 2018, fasc. 1, p. 1 s.

GALLUCCIO, Alessandra. La grande sezione della Corte di Giustizia si pronuncia sulle attese questioni pregiudiziali in materia di ne bis in idem, em Dir. pen. cont., ed. on-line del 21 marzo 2018, disponibile in http://www.penalecontemporaneo. it, ultimo accesso il 24 agosto 2018.

GAMBINI, Rosanna. Armonizzazione dei diritti nazionali nel segno della giurisprudenza europea, em Dir. pen. proc., 2009, p. 1169 s.

GITTARDI, Irene. La miccia è accesa: la Corte di cassazione fa diretta applicazione dei principi della Carta di Nizza in materia di ne bis in idem, em Dir. pen. cont., ed. on-line del 7 aprile 2017, p. 1 ss., disponibile in http://www.penalecontemporaneo. it, ultimo accesso il 24 agosto 2018.

GUARNIERI, Giuseppe. Regiudicata (diritto processuale penale), em Noviss. d. it., vol. XV, Torino: Utet, 1968, p. 231 s.

KOSTORIS, Roberto E. La tutela dei diritti fondamentali, em KOSTORIS, Roberto E. (a cura di), Manuale di procedura penale europea, Torino: Giappichelli, 2017, p. 84 s.

LAVARINI, Barbara. Il ne bis in idem convenzionale e "doppio binario" sanzionatorio: il problema del “doppio giudicato", em Leg. pen., ed. on-line, 14 marzo 2016, disponibile in http://www.legislazionepenale.it, ultimo accesso il 24 agosto 2018. LAVARINI, Barbara. Corte europea dei diritti umani e ne bis in idem: la crisi del “doppio binario" sanzionatorio, em Dir. pen. proc., 2016, p. 85 s. 
LAVARINI, Barbara. Il 'fatto' ai fini del ne bis in idem nella legge italiana e nella CEDU: la Corte costituzionale alla ricerca di un difficile equilibrio, in Proc. pen. giust., 2017 , fasc. 1 , p. $60 \mathrm{~s}$.

LOZZI, Gilberto. Profili di una indagine sui rapporti tra «ne bis in idem» e concorso formale di reati, Milano: Giuffrè, 1974.

LOZZI, Gilberto. Lezioni di procedura penale, Torino: Giappichelli, 2016.

MANACORDA, Stefano. Equazioni complesse: il ne bis in idem "ancipite" sul "doppio binario" per gli abusi di mercato al vaglio della giurisprudenza, em Dir. pen. proc., 2017, p. 520 s.

MANCUSO, Enrico Maria. Il giudicato nel processo penale, em UBERTIS, Giulio, VOENA, Giovanni Paolo (diretto da). em Trattato di procedura penale, vol. XLI.1, Milano: Giuffrè, 2012.

MANCUSO, Enrico Maria. Art. 4 prot. 7 - Divieto di secondo giudizio, em UBERTIS, Giulio, VIGANÒ, Francesco (a cura di). Corte di Strasburgo e giustizia penale, Torino: Giappichelli, 2016, p. 374 s.

MANCUSO, Enrico Maria. Ne bis in idem e giustizia sovranazionale, em GIARDA, Angelo, PERINI, Andrea, VARRASO, Gianluca (a cura di). La nuova giustizia penale tributaria, Padova: Cedam, 2016, p. 533 s.

MANES, Vittorio. Il giudice nel labirinto. Profili delle intersezioni tra diritto penale e fonti sovranazionali, Roma: Dike, 2012, p. 135 s.

MANTOVANI, Ferrando. Diritto penale, Padova: Cedam, 2017.

MAZZA, Oliviero. L'insostenibile convivenza fra ne bis in idem europeo e doppio binario sanzionatorio per i reati tributari, em Rass. Trib., 2015, fasc. 4, p. 1035.

MAZZA, Oliviero. Giustizia penale in trasformazione: profili d'indagine, em Arch. pen., 2012, fasc. 1, p. 17 s.

MONTAGNA, Mariangela. Ne bis in idem e sentenza non irrevocabile: si allarga l'ambito della preclusione, em Dir. pen. proc., 2005, p. 134 s.

NASCIMBENE, Bruno. Ne bis in idem, diritto internazionale, diritto europeo, em Eurojus, ed. on-line del 22 marzo 2018, disponibile in https://www.eurojus.it, ultimo accesso il 24 agosto 2018

PAGLIARO, Antonio. Fatto (dir. proc. pen), em Enc. dir., XVI, Milano: Giuffrè, 1967, p. 962 s.

PAULESU, Pier Paolo. Ne bis in idem e conflitti di giurisdizione, em KOSTORIS, Roberto E. (a cura di), Manuale di procedura penale europea, Torino: Giappichelli, 2017, p. 478 s. 
PISAPIA, Alice. Riflessioni sul principio del ne bis in idem alla luce delle recenti pronunce della Corte di Giustizia dell'Unione europea, em Cass. pen., 2013, p. 3272 s. PROCACCINO, Angela. Il ne bis in idem dalla "certezza del diritto" alla certezza del “diritto soggettivo", em GAITO, Alfredo, CHINNICI, Daniela (a cura di). Regole europee e processo penale, Padova: Cedam, 2016, p. 269 s.

PULITANÒ, Domenico. La Corte costituzionale sul ne bis in idem, em Cass. pen., 2017, p. 70 s.

PULITANÒ, Domenico. Ne bis in idem. Novità dalla Corte e problemi aperti, em Dir. pen. proc., 2016, p. 1588 s.

QUATTROCOLO, Serena. Riqualificazione del fatto nella sentenza penale e tutela del contraddittorio, Napoli: Jovene, 2011.

RAFARACI, Tommaso. Le nuove contestazioni nel processo penale, Milano: Giuffrè, 1996.

RAFARACI, Tommaso. Ne bis in idem, in Enc. dir., Annali, vol. III, Milano: Giuffrè, 2010, p. 857 s.

RAFARACI, Tommaso. Ne bis in idem e conflitti di giurisdizione in materia penale nello spazio di libertà, sicurezza e giustizia dell'Unione Europea, em Riv. dir. proc., 2007, p. 625 s.

RANALDI, Gaetano, GAITO, Federico. Introduzione allo studio dei rapporti tra ne bis in idem sostanziale e processuale, em Arch. pen., 2017, fasc. 1, p. 103 s.

RIVELLO, Pier Paolo. Analisi in tema di ne bis in idem, em Riv. it. dir. proc. pen., 1991, p. $500 \mathrm{~s}$.

RIVELLO, Pier Paolo. La nozione di "fatto" ai sensi dell'art. 649 c.p.p. e le perduranti incertezze interpretative ricollegabili al principio del ne bis in idem, em Riv. it. dir. proc. pen., 2014, p. 1411 s.

RIVELLO, Pier Paolo. I rapporti tra giudizio penale e tributario ed il rispetto del principio del ne bis in idem, em Dir. pen. cont., 2018, fasc. 1, p. 101 ss., disponibile in https://www.penalecontemporaneo.it, ultimo accesso il 24 agosto 2018.

SANTORIELLO, Ciro. Il ne bis in idem nella giurisprudenza della Corte di Giustizia UE: "così è se vi pare" (o almeno se così riterrà il giudice nazionale), em Il Penalista, ed. on-line del 23 aprile 2018, disponibile in https://www.ilpenalista.it, ultimo accesso il 24 agosto 2018.

SCOLETTA, Marco. Ne bis in idem e doppio binario in materia tributaria: legittimo sanzionare la società e punire il rappresentante legale per lo stesso fatto, em Dir. pen. 
cont., ed. on-line, 10 aprile 2017, disponibile in https://www.penalecontemporaneo.it, ultimo accesso il 24 agosto 2018.

UBERTIS, Giulio. Fatto e valore nel sistema probatorio penale, Milano: Giuffrè, 1979.

UBERTIS, Giulio. Principi di procedura penale europea. Le regole del giusto processo, Milano; Cortina, 2009.

UBERTIS, Giulio. Sistema multilivello dei diritti fondamentali e prospettiva abolizionista del processo contumaciale contumaciale (2009), em UBERTIS, Giulio. Argomenti di procedura penale, vol. III, Milano: Giuffrè, 2011, p. 185 s.

UBERTIS, Giulio. Equità e proporzionalità versus legalità processuale: eterogenesi dei fini?, em Arch. pen., 2017, fasc. p. 1 s.

UBERTIS, Giulio. Sistema di procedura penale, I, Principi generali, Milano: Giuffrè, 2017, p. 134.

VERVAELE, John. Ne bis in idem: verso un principio costituzionale transnazionale in UE?, em Riv. it. dir. proc. pen., 2014, p. 53 s.

VIGANÒ, Francesco. Doppio binario sanzionatorio e ne bis in idem: verso una diretta applicazione dell'art. 50 della Carta?, em Dir. pen. cont. - Riv. trim., 2014, n. 3-4, p. 234 s. disponibile in https://www.penalecontemporaneo.it, ultimo accesso il 24 agosto 2018.

VIGANÒ, Francesco. Art. 4 Prot. n. 7 - Diritto a non essere giudicato o punito due volte, em UBERTIS, Giulio, VIGANÒ, Francesco (a cura di). Corte di Strasburgo e giustizia penale e Corte di Strasburgo, Giappichelli, 2016, p. 383 s.

VIGANÒ, Francesco. A never-ending story? Alla Corte di Giustizia dell'Unione Europea la questione della compatibilità tra ne bis in idem e doppio binario sanzionatorio in materia, questa volta di abusi di mercato, em Dir. pen. cont., ed. on-line, 17 ottobre 2016, disponibile in https://www.penalecontemporaneo.it, ultimo accesso il 24 agosto 2018.

VIGANÒ, Francesco. La Grande camera della Corte di Strasburgo su ne bis in idem e doppio binario sanzionatorio, em Dir. pen. cont., ed. on-line, 18 novembre 2016, disponibile in https://www.penalecontemporaneo.it, ultimo accesso il 24 agosto 2018

VIGANÒ, Francesco. Una nuova sentenza di Strasburgo su ne bis in idem e reati tributari, em Dir. pen. cont., ed. on-line, 22 maggio 2017, disponibile in https:// www.penalecontemporaneo.it, ultimo accesso il 24 agosto 2018.

ZAGREBELSKY, Vladimiro. Le sanzioni Consob, l'equo processo e il ne bis in idem nella C.e.d.u., in Giur. it., 2014, p. 1199 s. 


\section{Informações adicionais e declarações dos autores (integridade científica)}

Declaração de conflito de interesses (conflict of interest declaration): o autor confirma que não há conflitos de interesse na realização das pesquisas expostas e na redação deste artigo.

Declaração de autoria e especificação das contribuições (declaration of authorship): todas e somente as pessoas que atendem os requisitos de autoria deste artigo estão listadas como autores.

Declaração de ineditismo e originalidade (declaration of originality): o autor assegura que o texto aqui publicado não foi divulgado anteriormente em outro meio e que futura republicação somente se realizará com a indicação expressa da referência desta publicação original; também atesta que não há plágio de terceiros ou autoplágio.

\section{Dados do processo editorial}

(http://www.ibraspp.com.br/revista/index.php/RBDPP/about/editorialPolicies)

- Recebido em: 16.07.2018

- Controle preliminar e verificação de plágio: 17.07.2018

- Avaliação 1: 13.08.2018

- Avaliação 2: 14.08.2018

- Decisão editorial preliminar: 18.07.2018

- Retorno rodada de correções 1: 06.09.2018

- Decisão editorial final: 29.09.2018
Equipe editorial envolvida

- Editor-chefe: 1 (VGV)

- Editores-associados: 2 (BC - FC)

- Revisores: 2

\section{COMO CITAR ESTE ARTIGO:}

CASSIBBA, Fabio Salvatore. I limiti oggettivi del ne bis in idem in Italia tra fonti nazionali ed europee. Revista Brasileira de Direito Processual Penal, Porto Alegre, vol. 4, n. 3, p. 953-1002, set./dez. 2018. https://doi.org/10.22197/rbdpp.v4i3.186

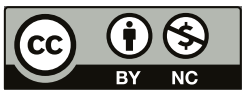

Esta obra está licenciada com uma Licença Creative Commons Atribuição-NãoComercial 4.0 Internacional. 